\title{
Coding Theorems for a (2,2)-Threshold Scheme with Detectability of Impersonation Attacks
}

\author{
Mitsugu Iwamoto, Member, IEEE, Hiroki Koga, Member, IEEE, \\ and Hirosuke Yamamoto, Fellow, IEEE
}

\begin{abstract}
Coding theorems on a (2,2)-threshold scheme with an opponent are discussed in an asymptotic setup, where the opponent tries to impersonate one of the two participants. A situation is considered where $n$ secrets $S^{n}$ from a memoryless source is blockwisely encoded to two shares and the two shares are decoded to $S^{n}$ with permitting negligible decoding error. We introduce correlation level of the two shares and characterize the minimum attainable rates of the shares and a uniform random number for realizing a $(2,2)$-threshold scheme that is secure against the impersonation attack by the opponent. It is shown that, if the correlation level between the two shares equals to an $\ell \geq 0$, the minimum attainable rates coincide with $H(S)+\ell$, where $H(S)$ denotes the entropy of the source, and the maximum attainable exponent of the success probability of the impersonation attack equals to $\ell$. It is also shown that a simple scheme using an ordinary $(2,2)$-threshold scheme attains all the bounds as well.
\end{abstract}

Index Terms-Correlated sources, hypothesis testing, impersonation attack, secret sharing scheme, threshold scheme.

\section{INTRODUCTION}

\section{A. Background and Motivations}

A secret sharing scheme [1], [2] is a well-known cryptographic technique that enables us to share a secret data among users. In $(t, m)$-threshold schemes, for example, a secret $S$ is encoded to $m$ shares, and the $m$ shares are distributed to respective participants. Any $t$ out of $m$ participants can recover $S$, while $t-1$ or fewer participants cannot obtain any information on $S$ in the sense of unconditional security.

In this paper, we focus on the secret sharing scheme in the presence of opponents. The objective of the opponents is cheating honest participants. That is, the opponents forge their shares and try to cheat the honest participants by injecting the forged shares in the recovery phase of $S$. This problem was

This paper is presented in part at IEEE International Symposium on Information Theory 2009, and IEEE Information Theory Workshop 2009. The work of M. Iwamoto is partially supported by the MEXT Grant-in-Aid for Young Scientists (B) No. 20760236 and No. 23760330. The work of H. Koga is supported in part by Grant-in-Aid from the Telecommunications Advancement Foundation. Copyright (c) 2011 IEEE. Personal use of this material is permitted. However, permission to use this material for any other purposes must be obtained from the IEEE by sending a request to pubspermissions@ieee.org.

M. Iwamoto is with the Center of Frontier Science and Engineering, University of Electro-Communications, 1-5-1 Chofugaoka, Chofu-shi, Tokyo 182-8585, Japan (e-mail: mitsugu@inf.uec.ac.jp).

H. Koga is with Tsukuba University, 1-1-1, Tennoudai, Tsukuba-shi, 3058577 Japan (e-mail: koga@iit.tsukuba.jp).

H. Yamamoto is with Graduate School of Frontier Sciences, University of Tokyo, 5-1-5 Kashiwanoha, Kashiwa-shi, Chiba 277-8561, Japan (e-mail: Hirosuke@ieee.org) firstly discussed by McEliece-Sarwate [3] and Karnin-GreeneHellman [4] from the viewpoint of error-correcting codes. In particular, Karnin-Greene-Hellman [4] and Tompa-Woll [5] clarified that it is impossible to detect cheating in Shamir's secret sharing scheme [1]. In addition, a construction of a cheating-detectable secret sharing scheme is proposed in [5] as an extension of Shamir's secret sharing scheme although it is much inefficient. So far several schemes have been proposed to overcome such disadvantages [6]-[9]. In particular, OgataKurosawa-Stinson [8] derived a lower bound on sizes of shares under a given maximum success probability $\varepsilon$ of cheating and the lower bound is attained if and only if a difference set exists.

In cheating-detectable threshold schemes, the shares must satisfy unforgeablity as well as the ordinary requirements as a threshold scheme. We can actually consider two types of attacks, impersonation attacks and substitution attacks, similarly to the attacks against secret-key authentication systems [10]. In the impersonation attack, opponents intend to impersonate participants by injecting forged shares without using the legitimate shares. The impersonation attack is regarded as successful if the forged shares are accepted in a recovery phase of a secret. On the other hand, in the substitution attack, some of the participants are malicious and forge their shares by using their shares. The objective of the malicious participants is cheating honest participants who want to recover $S$ from their shares.

For instance, Figure $\square$ shows the two types of attacks against a $(2,2)$-threshold scheme with two shares $X$ and $Y$. We assume that the ordinary requirements as a $(2,2)$-threshold schemes, $H(S \mid X)=H(S \mid Y)=H(S)$ and $H(S \mid X Y)=0$, are satisfied. In Fig. [1(a) an opponent generates a forged share $\bar{X}$ without using $X$ and $Y$ and tries to impersonate participant 1 who have a share $X$. In Fig. I(b) a participant with $X$ forges $\bar{X}$ by using $X$, but not using $Y$. We assume that in both cases $\bar{X}$ is generated probabilistically. Then, it is important to notice that, $\bar{X}$ is independent of $(X, Y)$ in Fig. 1(a), while $Y, X$ and $\bar{X}$ form a Markov chain in this order in Fig. 1(b). Thus, considering the two types of attacks against threshold schemes corresponds to giving two kinds of probabilistic structures for all the shares including the forged share.

Cheating-detectable secret sharing schemes are usually designed to detect substitution attacks [5]-[9] in a nonasymptotic setup, i.e., the decoding error is not allowed and the block coding is not considered. These studies treat the case where a coalition of more than one malicious participants generates forged shares. However, there exist the following 


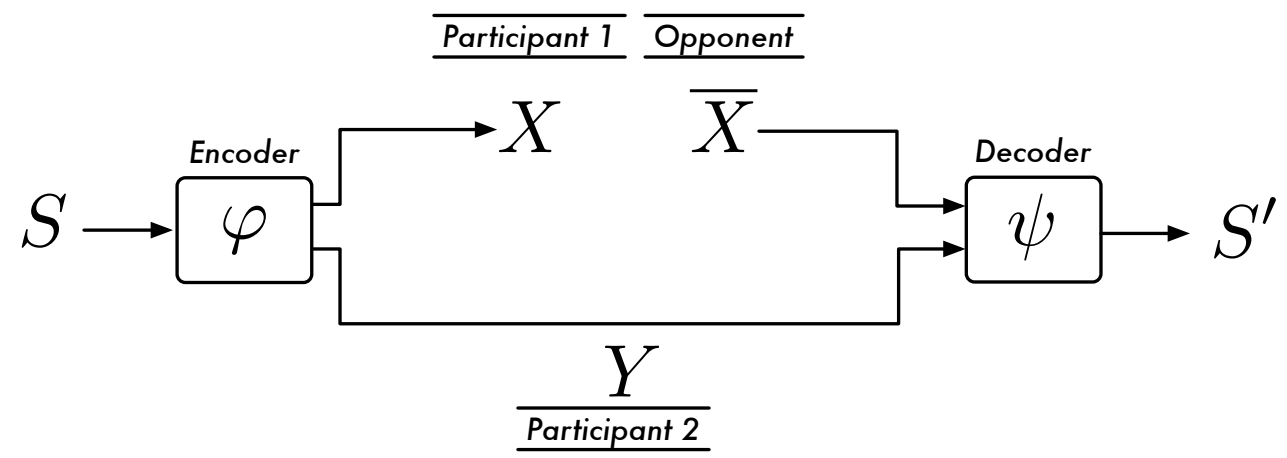

(a) A $(2,2)$-threshold scheme with an opponent who impersonates a participant who has a share $X$ (impersonation attack)

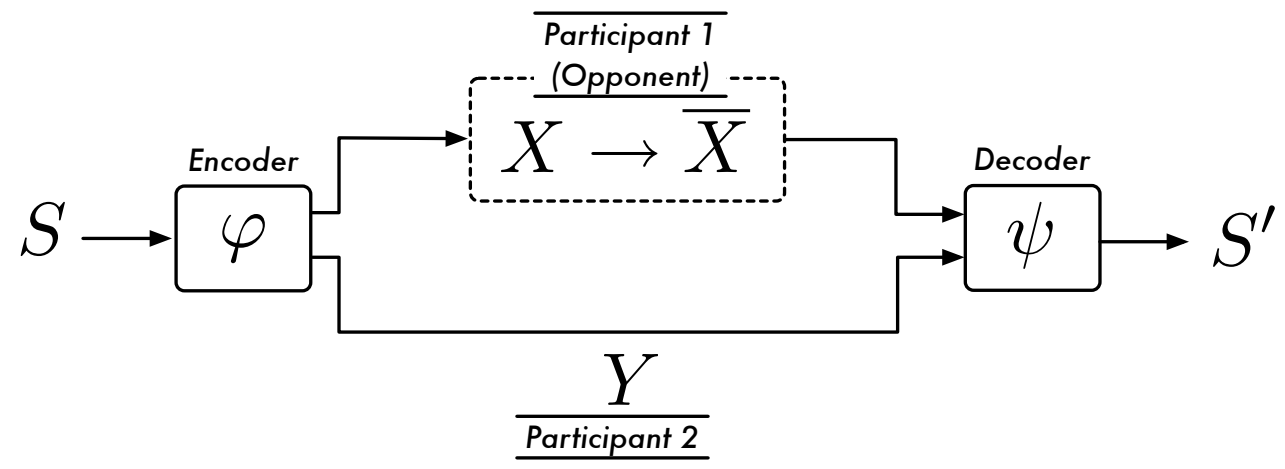

(b) A $(2,2)$-threshold scheme with an opponent who substitutes a share $\bar{X}$ for $X$ (substitution attack)

Fig. 1. Two (2,2)-threshold schemes with an opponent

drawbacks in cheating-detectable secret sharing schemes:

- According to [8], it is easy to derive the lower bounds of share rates, i.e., information bits per secret needed to describe shares, under a given success probability of cheating. Unfortunately, however, this result implies that the optimal share rates increase in order at least $1 / \varepsilon$ as $\varepsilon \rightarrow 0$, and hence, an arbitrarily small success probability of cheating cannot be realized with fixed finite share rates.

- An extension of Shamir's $(t, m)$-threshold scheme in [5] can detect both substitution and impersonation attacks. This scheme is simple but inefficient from the viewpoint of share sizes. In addition, the optimal construction [8] is based on a combinatoric structure called a difference set, where the difference set exists only in limited cases and therefore restricts sizes of a secret and shares. Hence, even in a $(2,2)$-threshold scheme, we cannot apply the optimal scheme to a secret $S$ of arbitrarily given size.

- Almost all constructions include the assumption that a secret is generated subject to a uniform probability distribution. This means that developing a near-optimum cheating-detectable secret sharing scheme for a secret subject to a non-uniform becomes another problem [9].

In this paper, we focus on the impersonation attack against a $(2,2)$-threshold scheme. Since the impersonation attack is weaker than the substitution attack, the impersonation attack is rarely discussed especially in the framework of secret sharing schemes. However, if we discuss the threshold scheme secure against impersonation attack in a certain asymptotic setup, we can unveil another information-theoretic aspect. In fact, we can find connections to hypothesis testing, authentication codes and Shannon's cipher system. In a practical point of view, we can consider a situation where impersonation attack seems to be valid. Suppose that in a $(2,2)$-threshold scheme one of the shares, say $X$, is a uniform random number that is independent of a secret $S$. In this case, the participants having $X$ may generate $\bar{X}$ subject to a distribution close to the uniform distribution because analysis of $X$ gives almost no information to the participant.

\section{B. Contribution of This Study}

In this paper, we formulate the problem of a threshold scheme secure against impersonation attacks in Shannontheoretic asymptotic setup [11], [12], and unveil new features included in the problem. We consider a situation where $n$ secrets that are generated from a discrete memoryless source are blockwisely encoded to two shares and the two shares are decoded to $n$ secrets with permitting negligible decoding error. While we consider impersonation attacks, the asymptotic $(2,2)$-threshold scheme treated in this paper has the following features which resolve the three drawbacks pointed above in cheating-detectable secret sharing schemes:

- An exponentially small success probability of impersonation attack is realized under finite share rates if the blocklength is sufficiently large. 


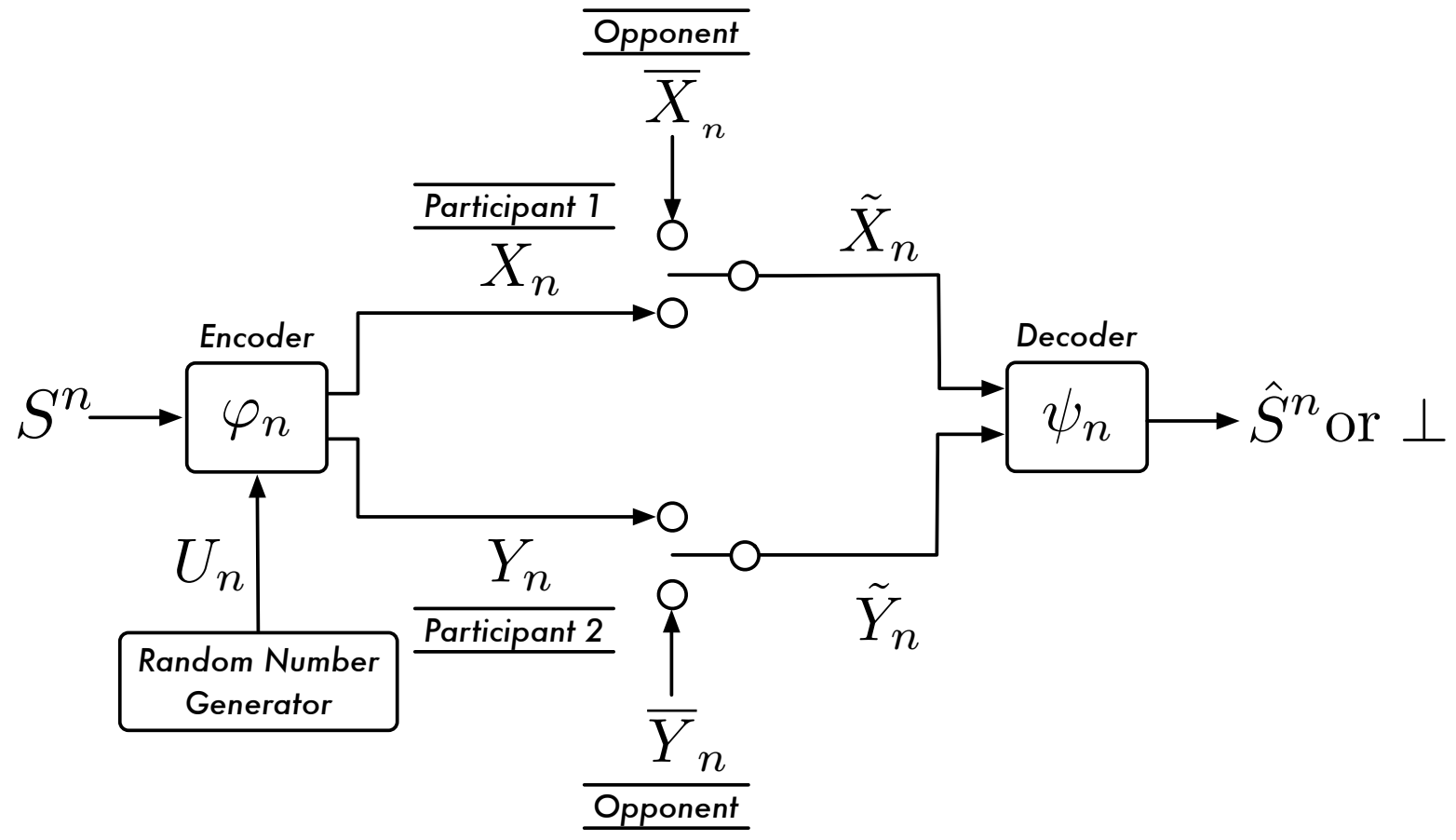

Fig. 2. A system model of $(2,2)$-threshold scheme with detectability of impersonation attacks

- The scheme uses no combinatoric structure and is applicable to arbitrary size of a secret.

- The probability distribution of a secret is arbitrary. In addition, the scheme can be applied to a more general class of sources.

Specifically, we give coding theorems on the $(2,2)$ threshold scheme for two cases of blockwise encoding and symbolwise encoding. In both cases we are interested in the minimum attainable rates for not only the two shares but also the uniform random number needed to a dealer for realizing a cheating-detectable $(2,2)$-threshold scheme in an asymptotic sense. We also evaluate the maximum attainable exponent of the success probability of the impersonation attack. It turns out that, if the two shares are correlated, we can easily realize the $(2,2)$-threshold scheme in an asymptotic sense that is secure against the impersonation attack. This fact motivates us to define a notion of correlation level of the two shares as the limit of the normalized mutual information between the two shares. In a non-asymptotic setup, we note that correlated shares are firstly discussed in [13] based on a combinatorial argument.

In the case of blockwise encoding, we consider an encoder that encodes $n$ secrets $S^{n}=S_{1} S_{2} \cdots S_{n}$ blockwisely to two shares $X_{n}$ and $Y_{n}$ by using a uniform random number $U_{n}$, where throughout the paper the superscript $n$ denotes the length and the subscripts $n$ indicate dependency of $n$. The two shares $X_{n}$ and $Y_{n}$ are decoded to $S^{n}$ with decoding error probability $P_{n}^{e}$ that satisfies $P_{n}^{e} \rightarrow 0$ as $n \rightarrow \infty$. The two shares are required to satisfy the security criteria $I\left(S^{n} ; X_{n}\right) / n \rightarrow 0$ and $I\left(S^{n} ; Y_{n}\right) / n \rightarrow 0$ as $n \rightarrow \infty$, where $I(\cdot ; \cdot)$ denotes the mutual information. We can prove that, if the correlation level of the shares is equal to $\ell$, none of the rates of $X_{n}, Y_{n}$ and $U_{n}$ cannot be less than $H(S)+\ell$, where $H(S)$ denotes the entropy of the source, and the exponent of the success probability of impersonation attack cannot be greater than $\ell$ (converse part). Furthermore, we can prove the existence of a symbolwise of pairs of an encoder and a decoder that attains all the bounds shown in the converse part (direct part). Both claims of the direct and the converse parts are easily extended to the case where $S^{n}$ is generated from a stationary ergodic source.

In the case of symbolwise encoding, we consider an encoder that encodes $n$ secrets $S^{n}$ to two shares $X^{n}=X_{1} X_{2} \cdots X_{n}$ and $Y^{n}=Y_{1} Y_{2} \cdots Y_{n}$ of length $n$ by using $n$ uniform random numbers $U^{n}=U_{1} U_{2} \cdots U_{n}$. In fact, $X^{n}$ and $Y^{n}$ are generated by $\left(X_{i}, Y_{i}\right)=f\left(S_{i}, U_{i}\right)$ for $i=1,2, \ldots, n$, where $f$ is an arbitrary deterministic encoder of an ordinary $(2,2)-$ threshold scheme satisfying $H\left(S_{i} \mid X_{i}\right)=H\left(S_{i} \mid Y_{i}\right)=H\left(S_{i}\right)$ and $H\left(S_{i} \mid X_{i} Y_{i}\right)=0$. Denote by $g$ a deterministic map satisfying $S_{i}=g\left(X_{i}, Y_{i}\right)$. We choose an appropriate $f$ so that $\left(X_{i}, Y_{i}\right), i=1,2, \ldots, n$, can be regarded as i.i.d. correlated random variables. It is shown that we can realize a $(2,2)-$ threshold scheme in an asymptotic sense in which $P_{n}^{e}$ vanishes as $n \rightarrow \infty, X^{n}$ and $Y^{n}$ satisfy a stronger requirement on the secrecy $I\left(S^{n} ; X^{n}\right)=I\left(S^{n} ; Y^{n}\right)=0$ and the exponent of the success probability of the impersonation attack is optimal. In the proof we construct a decoder of $X^{n}$ and $Y^{n}$ by using $g$ and a one-sided test for verifying the joint typicality of $X^{n}$ and $Y^{n}$. This kind of symbolwise setup is first discussed in [14] for authentication code. 


\section{Related Works, Organization}

The $(2,2)$-threshold scheme secure against the impersonation attack is motivated from the Shannon-theoretic authentication codes [10], [14]-[16]. In particular, in [14] the authors discuss the maximum attainable error exponent on the success probability of the impersonation attack subject to the vanishing decoding error probability. However, the results given in this paper is more involved. In fact, in the framework of $(2,2)$ threshold schemes we need to guarantee secrecy of a secret given one of the two shares. In addition, in this paper we succeeded in obtaining not only such a maximum exponent but also the minimum attainable sizes of the shares and the uniform random number.

The $(2,2)$-threshold scheme with detectability of impersonation attacks with the blockwise encoder can be viewed as one version of Shannon's cipher system ( [17]-[21] etc.) when one of the shares is an output from a random number generator. In a simple asymptotic setup of Shannon's cipher system [20], $n$ plaintexts $S^{n}$ generated from a memoryless source are encrypted to a cryptogram $W_{n}$ under a key $U_{n}$ and $W_{n}$ is decrypted to $S^{n}$ under the same key $U_{n}$ with permitting decoding error probability $P_{n}^{e}$. The encoder and the decoder are required to satisfy $P_{n}^{e} \rightarrow 0$ and $I\left(S^{n} ; W_{n}\right) / n \rightarrow 0$ as $n \rightarrow \infty$. In this setup, the minimum attainable rates of the cryptogram and the key coincide with the entropy $H(S)$ of the plaintext. The coding theorems given in this paper imply the same result under an additional requirement such that the correlation level of $W_{n}$ and $U_{n}$ is equal to zero, i.e., $\ell=0$.

The $(2,2)$-threshold scheme with detectability of impersonation attacks with the symbolwise encoder is related to the problem of secret key agreement [21], [22]. In the secret key agreement problem of the source type model [22], two users have $n$ outputs $X^{n}=X_{1} X_{2} \cdots X_{n} \in \mathcal{X}^{n}$ and $Y^{n}=Y_{1} Y_{2} \cdots Y_{n} \in \mathcal{Y}^{n}$ from two correlated memoryless source, respectively, where $\left(X_{i}, Y_{i}\right), i=1,2, \ldots, n$ are i.i.d. copies of $(X, Y) \in \mathcal{X} \times \mathcal{Y}$ subject to a joint probability distribution $P_{X Y}$. The two user try to share a nearly uniform random number with the maximum rate $I(X ; Y)$ by public communications. On the contrary, the symbolwise encoder in the $(2,2)$-threshold scheme with detectability of impersonation attacks can be interpreted as a generator of correlated random variables $X^{n}=X_{1} X_{2} \cdots X_{n} \in \mathcal{X}^{n}$ and $Y^{n}=Y_{1} Y_{2} \cdots Y_{n} \in \mathcal{Y}^{n}$ given independent random variables $S^{n}$ and $U^{n}$, where $\left(X_{i}, Y_{i}\right), i=1,2, \ldots, n$, are regarded as $n$ i.i.d. copies of $(X, Y) \sim P_{X Y}$. Since the correlation level $X^{n}$ and $Y^{n}$ coincides with $I(X ; Y)$, the minimum attainable rate of $U^{n}$ turns out to be $H(S)+I(X ; Y)$. That is, we need an extra cost of $I(X ; Y)$ in order to generate correlated two shares.

The rest of this paper is organized as follows: In Section II, a $(2,2)$-threshold scheme with detectability of impersonation attacks with correlation level $\ell$ in an asymptotic setup is formulated. The coding theorems for the blockwise encoder are given in Section $\mathbb{I I I}$. Section $\mathbb{\mathbb { V }}$ is devoted to the proofs of the coding theorems. A construction of encoders and decoders based on a non-asymptotic (2,2)-threshold scheme and its optimality are discussed in Section $\nabla$.

\section{Problem Setting}

We consider a $(2,2)$-threshold scheme depicted in Fig. \. Assume for an integer $n \geq 1$ that a source generates an $n-$ tuple of secrets $S^{n}=S_{1} S_{2} \cdots S_{n}$ independently subject to a probability distribution $P_{S}$ on a finite set $\mathcal{S}$. Denote by $P_{S^{n}}$ the probability distribution of $S^{n}$ induced by $P_{S}$, and let $P_{S^{n}}\left(s^{n}\right)$ be the probability that $S^{n}=s^{n}$ for an $s^{n} \in \mathcal{S}^{n}$. Since the source is memoryless, it holds that $P_{S^{n}}\left(s^{n}\right)=\prod_{i=1}^{n} P_{S}\left(s_{i}\right)$ for all $n \geq 1$ where $s^{n}=s_{1} s_{2} \cdots s_{n}$.

In Fig. \, let $U_{n}$ be the random variable subject to the uniform distribution on a finite set $\mathcal{U}_{n}$. Assume that $U_{n}$ is independent of $S^{n}$. In this paper, we use the subscript $n$ to indicate dependency of $n$, while the superscript $n$ implies the length. We denote by $P_{U_{n}}$ a probability distribution of $U_{n}$, i.e., it holds that $P_{U_{n}}\left(u_{n}\right)=1 /\left|\mathcal{U}_{n}\right|$ for all $u_{n} \in \mathcal{U}_{n}$ where $|\cdot|$ denotes the cardinality.

An encoder is defined as a deterministic map $\varphi_{n}: \mathcal{S}^{n} \times$ $\mathcal{U}_{n} \rightarrow \mathcal{X}_{n} \times \mathcal{Y}_{n}$, where $\mathcal{X}_{n}$ and $\mathcal{Y}_{n}$ are finite sets in which shares $X_{n}$ and $Y_{n}$ take values, respectively. Hence, we can write

$$
\left(X_{n}, Y_{n}\right)=\varphi_{n}\left(S^{n}, U_{n}\right)
$$

from which we can see that $X_{n}$ and $Y_{n}$ are also random variables. The joint probability distribution $P_{X_{n} Y_{n}}$ of $X_{n}$ and $Y_{n}$ is induced from (II). The shares $X_{n}$ and $Y_{n}$ are distributed securely to participants 1 and 2, respectively.

Next, consider a situation where an opponent may impersonate one of the two participants. When the opponent impersonates participant 1 , the opponent behaves as if he/she were a participant 1 by injecting a forged share $\bar{X}_{n} \in \mathcal{X}_{n}$ instead of $X_{n}$. This attack is regarded as successful if a decoder fails to detect impersonation attacks and outputs an element of $\mathcal{S}^{n}$ from $\bar{X}_{n}$ and $Y_{n}$. Here, we assume that the opponent generates $\bar{X}_{n}$ without using $X_{n}$. According to [10], [14]-[16], such attack is called impersonation attack as opposed to substitution attack. Similarly, in the case of deceiving participant 1 , the opponent forges a share $\bar{Y}_{n}$ without using $Y_{n}$, and tries to impersonate participant 2 . In this case, the attack succeeds when the decoder outputs an element of $\mathcal{S}^{n}$ from $X_{n}$ and $\bar{Y}_{n}$. Summarizing, letting $\tilde{X}_{n}$ and $\tilde{Y}_{n}$ be the inputs to a decoder, the following three cases must be considered:

A decoder is defined as a deterministic map $\psi_{n}: \mathcal{X}_{n} \times$ $\mathcal{Y}_{n} \rightarrow \mathcal{S}^{n} \cup\{\perp\}$, where $\perp$ is a symbol to declare the detection of an impersonated attack, i.e., (a1) or (a2). We note here that the decoder cannot know in advance which one of $(\mathrm{a} 0)$ (a2) actually occurs. On the other hand, we assume that the opponent knows everything about the encoder and the decoder except for realizations of $S^{n}, U_{n}, X_{n}$ and $Y_{n}$.

In this situation, we define success probabilities of impersonation attacks. Let $\mathcal{A}_{n} \subset \mathcal{X}_{n} \times \mathcal{Y}_{n}$ be the region that the decoder $\psi_{n}$ accepts the pair of shares $\left(\tilde{X}_{n}, \tilde{Y}_{n}\right)$ and outputs an element of $\mathcal{S}^{n}$, i.e.,

$$
\mathcal{A}_{n}=\left\{\left(x_{n}, y_{n}\right) \in \mathcal{X}_{n} \times \mathcal{Y}_{n}: \psi_{n}\left(x_{n}, y_{n}\right) \in \mathcal{S}^{n}\right\} .
$$


Now, recall that the impersonation attack succeeds if the decoder outputs an element of $\mathcal{S}^{n}$ when one of (a1) and (a2) occurs. In the case of (a1), i.e., the opponent impersonates participant 1 , we note that he/she generates a forged share $\bar{X}_{n}$ according to a probability distribution $P_{\bar{X}_{n}}$ independently from $S^{n}, U_{n}, X_{n}$, and $Y_{n}$. In addition, the opponent tries to optimize $P_{\bar{X}_{n}}$ so that $\left(\bar{X}_{n}, Y_{n}\right)$ can be accepted by the decoder with the maximum probability. This motivates us to define a success probability to impersonate participant 1 by

$$
P_{n}^{X}=\max _{P_{\bar{X}_{n}}} \operatorname{Pr}\left\{\left(\bar{X}_{n}, Y_{n}\right) \in \mathcal{A}_{n}\right\}
$$

where the maximization of $P_{\bar{X}_{n}}$ is taken over all probability distributions on $\mathcal{X}_{n}$, and $\operatorname{Pr}\{\cdot\}$ means the probability with respect to the (joint) probability distribution of random variable(s) between the parentheses, i.e., $\left(\bar{X}_{n}, Y_{n}\right) \sim P_{\bar{X}_{n} Y_{n}}=$ $P_{\bar{X}_{n}} P_{Y_{n}}$ in this case. Similarly, the maximum success probability for the impersonation to participant 2 can be defined as

$$
P_{n}^{Y}=\max _{P_{\bar{Y}_{n}}} \operatorname{Pr}\left\{\left(X_{n}, \bar{Y}_{n}\right) \in \mathcal{A}_{n}\right\}
$$

where $\operatorname{Pr}\{\cdot\}$ is taken with respect to $\left(X_{n}, \bar{Y}_{n}\right) \sim P_{X_{n} \bar{Y}_{n}}=$ $P_{X_{n}} P_{\bar{Y}_{n}}$.

The decoding error occurs when $S^{n}$ is not correctly decoded from legitimate shares in the case of $(\mathrm{a} 0)$. Hence, the decoding error probability can be written as

$$
P_{n}^{e}=\operatorname{Pr}\left\{\psi_{n}\left(\varphi_{n}\left(S^{n}, U_{n}\right)\right) \neq S^{n}\right\} .
$$

It is easy to see that if $\left(x_{n}, y_{n}\right) \notin \mathcal{A}_{n}$, then $\psi_{n}\left(x_{n}, y_{n}\right)=$ $\perp \notin \mathcal{S}^{n}$. Hence, we have

$$
P_{n}^{e} \geq \operatorname{Pr}\left\{\left(X_{n}, Y_{n}\right) \notin \mathcal{A}_{n}\right\}
$$

for any pair of an encoder $\varphi_{n}$ and a decoder $\psi_{n}$.

Now, we can define a $(2,2)$-threshold scheme in an asymptotic setup as follows:

Definition 1: We say that a sequence $\left\{\left(\varphi_{n}, \psi_{n}\right)\right\}_{n=1}^{\infty}$ of an encoder $\varphi_{n}$ and a decoder $\psi_{n}$ asymptotically realizes a $(2,2)$ threshold scheme if it satisfies

$$
\lim _{n \rightarrow \infty} P_{n}^{e}=0
$$

and

$$
\lim _{n \rightarrow \infty} \frac{1}{n} I\left(S^{n} ; X_{n}\right)=\lim _{n \rightarrow \infty} \frac{1}{n} I\left(S^{n} ; Y_{n}\right)=0
$$

where $I(\cdot ; \cdot)$ denotes the mutual information.

The condition (D) guarantees that the decoding error probability is negligible if the blocklength $n$ is sufficiently large. Note that Fano's inequality [23, Theorem 2.10.1] tells us that

$$
\frac{1}{n} H\left(S^{n} \mid X_{n} Y_{n}\right) \leq \frac{1}{n} h\left(P_{n}^{e}\right)+P_{n}^{e} \log |\mathcal{S}|
$$

where $\log (\cdot)=\log _{2}(\cdot)$ throughout the paper, and $H(\cdot \mid \cdot)$ and $h(\cdot)$ are the conditional and the binary entropies, respectively. Hence, if (D) is satisfied, then we have

$$
\lim _{n \rightarrow \infty} \frac{1}{n} H\left(S^{n} \mid X_{n} Y_{n}\right)=0
$$

due to the non-negativity of the conditional entropy. On the other hand, the condition (8) ensures that $S^{n}$ is secure against the leakage from one of $X_{n}$ and $Y_{n}$ if $n$ is sufficiently large. That is, $S^{n}$ and either one of the shares are almost independent under such a condition. We also note that, since $S^{n}$ is generated from a memoryless source, (区) implies that

$$
\lim _{n \rightarrow \infty} \frac{1}{n} H\left(S^{n} \mid X_{n}\right)=\lim _{n \rightarrow \infty} \frac{1}{n} H\left(S^{n} \mid Y_{n}\right)=H(S)
$$

where $H(\cdot)$ denotes the entropy.

We conclude this section with introducing a notion of correlation level. The mutual information of two shares plays a crucial role in detecting impersonation attacks, which will be clarified in the following sections.

Definition 2: Let $\left\{\left(X_{n}, Y_{n}\right)\right\}_{n=1}^{\infty}$ be a pair of shares generated by a sequence of encoders $\left\{\varphi_{n}\right\}_{n=1}^{\infty}$. Then, a non-negative number $\ell$ is said to be a correlation level of $\left\{\left(X_{n}, Y_{n}\right)\right\}_{n=0}^{\infty}$ if it holds that

$$
\lim _{n \rightarrow \infty} \frac{1}{n} I\left(X_{n} ; Y_{n}\right)=\ell
$$

In particular, if a sequence $\left\{\left(\varphi_{n}, \psi_{n}\right)\right\}_{n=1}^{\infty}$ of an encoder $\varphi_{n}$ and a decoder $\psi_{n}$ satisfies Definition $\square$ and the sequence of shares $\left\{\left(X_{n}, Y_{n}\right)\right\}_{n=0}^{\infty}$ generated from $\left\{\varphi_{n}\right\}_{n=1}^{\infty}$ satisfies (12), we say that $\left\{\left(\varphi_{n}, \psi_{n}\right)\right\}_{n=1}^{\infty}$ asymptotically realizes a $(2,2)$ threshold scheme with correlation level $\ell$.

Remark 1: Note that the sequence $\left\{I\left(X_{n} ; Y_{n}\right) / n\right\}_{n=1}^{\infty}$ in (122) does not have a limit in general if $\left\{\left(X_{n}, Y_{n}\right)\right\}_{n=1}^{\infty}$ is generated by an arbitrary sequence of encoders $\left\{\varphi_{n}\right\}_{n=1}^{\infty}$. Hence, (112) actually requires the existence of the limit for the sequence $\left\{I\left(X_{n} ; Y_{n}\right) / n\right\}_{n=1}^{\infty}$, and the limit equals to $\ell$.

\section{Coding TheOrems FOR A $(2,2)$-THRESHOLD SCHEME WITH DETECTABILITY OF IMPERSONATION ATTACKS}

In this section, we give coding theorems for $\left\{\left(\varphi_{n}, \psi_{n}\right)\right\}_{n=1}^{\infty}$ that asymptotically realizes a $(2,2)$-threshold scheme with correlation level $\ell$. We are interested in not only the rates of $X_{n}, Y_{n}$ and $U_{n}$ but also the exponents of $P_{n}^{X}$ and $P_{n}^{Y}$ of the sequence $\left\{\left(\varphi_{n}, \psi_{n}\right)\right\}_{n=1}^{\infty}$. The following theorem is the converse part of the coding theorem with respect to such rates and exponents.

Theorem 1: For any sequence $\left\{\left(\varphi_{n}, \psi_{n}\right)\right\}_{n=1}^{\infty}$ of an encoder $\varphi_{n}$ and a decoder $\psi_{n}$ that asymptotically realizes a $(2,2)-$ threshold scheme with correlation level $\ell$, it holds that

$$
\begin{aligned}
& \liminf _{n \rightarrow \infty} \frac{1}{n} \log \left|\mathcal{X}_{n}\right| \geq H(S)+\ell \\
& \liminf _{n \rightarrow \infty} \frac{1}{n} \log \left|\mathcal{Y}_{n}\right| \geq H(S)+\ell \\
& \liminf _{n \rightarrow \infty} \frac{1}{n} \log \left|\mathcal{U}_{n}\right| \geq H(S)+\ell
\end{aligned}
$$

and

$$
\limsup _{n \rightarrow \infty} \max \left\{-\frac{1}{n} \log P_{n}^{X},-\frac{1}{n} \log P_{n}^{Y}\right\} \leq \ell .
$$




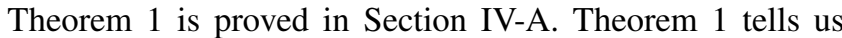
that for an arbitrarily small $\gamma>0$ the rates of $X_{n}, Y_{n}$ and $U_{n}$ cannot be less than $H(S)+\ell-\gamma$ for all sufficiently large $n$, where $\ell$ is an arbitrarily given correlation level. In fact, by noticing that $H(S)+\ell \geq H(S)$ for any $\ell \geq 0$, the bounds on the right hand sides of ([13)-(피) coincide with the bounds in [12, Theorem 1] for $(2,2)$-threshold schemes when $\ell=0$. Theorem $\mathrm{I}$ also indicates that the correlation level of shares is an upper bound on the exponents of $P_{n}^{X}$ and $P_{n}^{Y}$.

The direct part of the coding theorem corresponding to Theorem $\mathbb{D}$ is as follows:

Theorem 2: For an arbitrarily given non-negative number $\ell \geq 0$, there exists a sequence $\left\{\left(\varphi_{n}^{*}, \psi_{n}^{*}\right)\right\}_{n=1}^{\infty}$ of an encoder $\varphi_{n}^{*}$ and a decoder $\psi_{n}^{*}$ that asymptotically realizes a $(2,2)$ threshold scheme with correlation level $\ell$ satisfying

$$
\begin{aligned}
& \limsup _{n \rightarrow \infty} \frac{1}{n} \log \left|\mathcal{X}_{n}\right| \leq H(S)+\ell \\
& \limsup _{n \rightarrow \infty} \frac{1}{n} \log \left|\mathcal{Y}_{n}\right| \leq H(S)+\ell \\
& \limsup _{n \rightarrow \infty} \frac{1}{n} \log \left|\mathcal{U}_{n}\right| \leq H(S)+\ell
\end{aligned}
$$

and

$$
\liminf _{n \rightarrow \infty} \min \left\{-\frac{1}{n} \log P_{n}^{X},-\frac{1}{n} \log P_{n}^{Y}\right\} \geq \ell .
$$

In particular, the above $\left\{\left(\varphi_{n}^{*}, \psi_{n}^{*}\right)\right\}_{n=1}^{\infty}$ also satisfies

$$
I\left(S^{n} ; X_{n}\right)=I\left(S^{n} ; Y_{n}\right)=0 \text { for all } n \geq 1
$$

which is stronger than the condition in ( $(\mathbb{8})$.

\section{The proof of Theorem $\square$ is given in Section $[\nabla-\mathrm{B}$.}

Remark 2: Theorem प guarantees that $\left\{\left(\varphi_{n}^{*}, \psi_{n}^{*}\right)\right\}_{n=1}^{\infty}$ in Theorem $\square$ attains the minimum rates of $X_{n}, Y_{n}$, and $U_{n}$, and the maximum exponents of $P_{n}^{X}$ and $P_{n}^{Y}$. Furthermore, the limits exist for these rates and exponents, i.e., it holds that

$$
\begin{aligned}
\lim _{n \rightarrow \infty} \frac{1}{n} \log \left|\mathcal{X}_{n}\right|=\lim _{n \rightarrow \infty} \frac{1}{n} \log \left|\mathcal{Y}_{n}\right| & =\lim _{n \rightarrow \infty} \frac{1}{n} \log \left|\mathcal{U}_{n}\right| \\
& =H(S)+\ell
\end{aligned}
$$

and

$$
\lim _{n \rightarrow \infty}-\frac{1}{n} \log P_{n}^{X}=\lim _{n \rightarrow \infty}-\frac{1}{n} \log P_{n}^{Y}=\ell .
$$

\section{Proofs of Theorems \AND $\square$}

This section is devoted to the proofs of Theorems $\square$ and ㅁ. In the proof of Theorem M, we use a relationship between hypothesis testing and the $(2,2)$-threshold scheme with detectability of impersonation attacks with correlation level $\ell$, which originates from [16] and developed by [14], [15].

\section{A. Proof of Theorem $\square$}

Fix $\ell \geq 0$ arbitrarily. We first prove ([13). From the basic properties of the entropy and the mutual information, it holds that

$$
\begin{aligned}
H\left(X_{n}\right) & =I\left(X_{n} ; Y_{n}\right)+H\left(X_{n} \mid Y_{n}\right) \\
& \geq I\left(X_{n} ; Y_{n}\right)+H\left(X_{n} \mid Y_{n}\right)-H\left(X_{n} \mid Y_{n} S^{n}\right) \\
& =I\left(X_{n} ; Y_{n}\right)+I\left(X_{n} ; S^{n} \mid Y_{n}\right) \\
& =I\left(X_{n} ; Y_{n}\right)+H\left(S^{n} \mid Y_{n}\right)-H\left(S^{n} \mid X_{n} Y_{n}\right) .
\end{aligned}
$$

Hence, ([13) is established because

$$
\begin{aligned}
\liminf _{n \rightarrow \infty} \frac{1}{n} \log \left|\mathcal{X}_{n}\right| \geq & \liminf _{n \rightarrow \infty} \frac{1}{n} H\left(X_{n}\right) \\
\geq & \liminf _{n \rightarrow \infty} \frac{1}{n} I\left(X_{n} ; Y_{n}\right) \\
& +\liminf _{n \rightarrow \infty} \frac{1}{n} H\left(S^{n} \mid Y_{n}\right) \\
& \quad-\limsup _{n \rightarrow \infty} \frac{1}{n} H\left(S^{n} \mid X_{n} Y_{n}\right) \\
= & \ell+H(S)
\end{aligned}
$$

where the last inequality and the equality are due to (24) and (미)-(피), respectively. We can establish (펴) in essentially the same way.

Next, we prove ([15). Since the encoder $\varphi_{n}$ is deterministic for each $n \geq 1$, we have

$$
\begin{aligned}
H\left(X_{n} Y_{n}\right) & \leq H\left(S^{n} U_{n}\right) \\
& =n H(S)+H\left(U_{n}\right)
\end{aligned}
$$

for all $n \geq 1$, where the equality follows because $S^{n}$ is independent of $U_{n}$ and is generated from a memoryless source. On the other hand, recalling that

$$
H\left(X_{n} Y_{n}\right)=H\left(X_{n}\right)+H\left(Y_{n}\right)-I\left(X_{n} ; Y_{n}\right)
$$

it follows from (26) and (27) that

$$
\begin{aligned}
& \frac{1}{n} \log \left|\mathcal{U}_{n}\right|=\frac{1}{n} H\left(U_{n}\right) \\
&=\frac{1}{n} H\left(X_{n} Y_{n}\right)-H(S) \\
& \geq \frac{1}{n}\left\{H\left(X_{n}\right)+H\left(Y_{n}\right)-I\left(X_{n} ; Y_{n}\right)\right\}-H(S) \\
& \quad \text { for all } n \geq 1
\end{aligned}
$$

where the first equality follows from the uniformity of $U_{n} \in$ $\mathcal{U}_{n}$. Therefore, we have

$$
\begin{aligned}
\liminf _{n \rightarrow \infty} \frac{1}{n} \log \left|\mathcal{U}_{n}\right| \geq & \liminf _{n \rightarrow \infty} \frac{1}{n} H\left(X_{n}\right)+\liminf _{n \rightarrow \infty} \frac{1}{n} H\left(Y_{n}\right) \\
& -\limsup _{n \rightarrow \infty} \frac{1}{n} I\left(X_{n} ; Y_{n}\right)-H(S) \\
\geq & H(S)+\ell
\end{aligned}
$$

where the last inequality follows from ([2) and ([25). Note that we have $\liminf _{n \rightarrow \infty} H\left(Y_{n}\right) \geq H(S)+\ell$ in the same way as (25).

To prove (16), we use the fact that the decoding error probability and the success probabilities of impersonation attack in a $(2,2)$-threshold scheme with correlation level $\ell$ are closely related to the error probabilities of the first kind 
and the second kind in hypothesis testing, respectively, which is pointed out in [14]-[16]. Let us consider a simple hypothesis test with the following two hypotheses:

$$
\begin{aligned}
& H_{0}:\left(\tilde{X}_{n}, \tilde{Y}_{n}\right) \sim P_{X_{n} Y_{n}} \\
& H_{1}:\left(\tilde{X}_{n}, \tilde{Y}_{n}\right) \sim P_{X_{n}} P_{Y_{n}} .
\end{aligned}
$$

Let $\mathcal{A}_{n} \subset \mathcal{X}_{n} \times \mathcal{Y}_{n}$ denote an acceptance region for the null hypothesis $H_{0}$. Then, the error probability of the first kind and the error probability of the second kind of the above hypothesis testing are given by

$$
\begin{aligned}
& \alpha_{n} \stackrel{\text { def }}{=} \sum_{\left(x_{n}, y_{n}\right) \in \mathcal{A}_{n}^{c}} P_{X_{n} Y_{n}}\left(x_{n}, y_{n}\right)=\operatorname{Pr}\left\{\left(X_{n}, Y_{n}\right) \notin \mathcal{A}_{n}\right\} \\
& \beta_{n} \stackrel{\text { def }}{=} \sum_{\left(x_{n}, y_{n}\right) \in \mathcal{A}_{n}} P_{X_{n}}\left(x_{n}\right) P_{Y_{n}}\left(y_{n}\right)
\end{aligned}
$$

where $\mathcal{A}_{n}^{c}$ denotes the complement set of $\mathcal{A}_{n}$. It is easy to see from (G) that $P_{n}^{e} \geq \alpha_{n}$ holds for any $n \geq 1$. Hence, in view of (प]), we have

$$
\lim _{n \rightarrow \infty} \alpha_{n}=0 .
$$

Furthermore, it follows from (B]) that

$$
\begin{aligned}
P_{n}^{X} & =\max _{P_{\bar{X}_{n}}} \operatorname{Pr}\left\{\left(\bar{X}_{n}, Y_{n}\right) \in \mathcal{A}_{n}\right\} \\
& =\max _{P_{\bar{X}_{n}}} \sum_{\left(x_{n}, y_{n}\right) \in \mathcal{A}_{n}} P_{\bar{X}_{n}}\left(x_{n}\right) P_{Y_{n}}\left(y_{n}\right) \\
& \geq \beta_{n} .
\end{aligned}
$$

Similarly, we also have $P_{n}^{Y} \geq \beta_{n}$. Therefore, it holds that

$$
\begin{array}{r}
-\frac{1}{n} \log \beta_{n} \geq \max \left\{-\frac{1}{n} \log P_{n}^{X},-\frac{1}{n} \log P_{n}^{Y}\right\} \\
\text { for all } n \geq 1 .
\end{array}
$$

According to [24, Theorem 4.4.1] and [14, Theorem 2], we have

$$
\begin{aligned}
I\left(X_{n} ; Y_{n}\right)=\sum_{\substack{\left(x_{n}, y_{n}\right) \\
\in \mathcal{X}_{n} \times \mathcal{Y}_{n}}} P_{X_{n} Y_{n}}\left(x_{n}, y_{n}\right) \log \frac{P_{X_{n} Y_{n}}\left(x_{n}, y_{n}\right)}{P_{X_{n}}\left(y_{n}\right) P_{Y_{n}}\left(y_{n}\right)} \\
=\sum_{\left(x_{n}, y_{n}\right) \in \mathcal{A}_{n}} P_{X_{n} Y_{n}}\left(x_{n}, y_{n}\right) \log \frac{P_{X_{n} Y_{n}}\left(x_{n}, y_{n}\right)}{P_{X_{n}}\left(x_{n}\right) P_{Y_{n}}\left(y_{n}\right)} \\
\quad+\sum_{\left(x_{n}, y_{n}\right) \in \mathcal{A}_{n}^{c}} P_{X_{n} Y_{n}}\left(x_{n}, y_{n}\right) \log \frac{P_{X_{n} Y_{n}}\left(x_{n}, y_{n}\right)}{P_{X_{n}}\left(x_{n}\right) P_{Y_{n}}\left(y_{n}\right)} \\
\geq \\
\left.\quad \sum_{\mathcal{A}_{n}} P_{X_{n} Y_{n}}\left(x_{n}, y_{n}\right)\right) \log \frac{\sum_{\mathcal{A}_{n}} P_{X_{n} Y_{n}}\left(x_{n}, y_{n}\right)}{\sum P_{\mathcal{A}_{n}} P_{X_{n}}\left(x_{n}\right) P_{Y_{n}}\left(y_{n}\right)} \\
\quad+\left(\sum_{\mathcal{A}_{n}^{c}} P_{X_{n} Y_{n}}\left(x_{n}, y_{n}\right)\right) \log \frac{\sum_{\mathcal{A}_{n}^{c}} P_{X_{n} Y_{n}}\left(x_{n}, y_{n}\right)}{\sum_{\mathcal{A}_{n}^{c}} P_{X_{n}}\left(x_{n}\right) P_{Y_{n}}\left(y_{n}\right)} \\
=\left(1-\alpha_{n}\right) \log \frac{1-\alpha_{n}}{\beta_{n}}+\alpha_{n} \log \frac{\alpha_{n}}{1-\beta_{n}} \\
=-h\left(\alpha_{n}\right)-\left(1-\alpha_{n}\right) \log \beta_{n}-\alpha_{n} \log \left(1-\beta_{n}\right) \\
\geq
\end{aligned}
$$

where the first inequality follows from the log sum inequality, and the second inequality holds because $-\alpha_{n} \log \left(1-\beta_{n}\right) \geq 0$. Hence, it follows from (B6) and (B7) that

$$
\begin{aligned}
\frac{1}{n} I\left(X_{n} ; Y_{n}\right) \geq & -\frac{h\left(\alpha_{n}\right)}{n} \\
& +\left(1-\alpha_{n}\right) \max \left\{-\frac{1}{n} \log P_{n}^{X},-\frac{1}{n} \log P_{n}^{Y}\right\} \\
\text { for all } n \geq 1 . & \text { (38) }
\end{aligned}
$$

Therefore, we have (1[6) by taking the limit superior of both sides of (B8) and noticing (B4).

Remark 3: The claim of Theorem $\square$ can be easily extended to the case where $S^{n}$ is generated from a stationary source. For the case of the stationary source, the entropy $H(S)$ in the statement of Theorem $\square$ is replaced with the entropy rate $H \stackrel{\text { def }}{=} \lim _{n \rightarrow \infty} H\left(S^{n}\right) / n$. By recalling the existence of the limit of $\left\{H\left(S^{n}\right) / n\right\}_{n=1}^{\infty}$ [23, Theorem 4.2.1], we can easily check that both the left hand sides of (25) and (29) are bounded by $H+\ell$.

\section{B. Proof of Theorem \]}

We choose arbitrarily a sequence $\left\{\gamma_{n}\right\}_{n=1}^{\infty}$ of positive numbers that satisfies $\lim _{n \rightarrow \infty} \gamma_{n}=0$ and $\lim _{n \rightarrow \infty} \sqrt{n} \gamma_{n}=\infty$. Let $\mathcal{T}_{\gamma_{n}}$ be the typical set defined by

$$
\mathcal{T}_{\gamma_{n}}=\left\{s^{n} \in \mathcal{S}^{n}:\left|\frac{1}{n} \log \frac{1}{P_{S^{n}}\left(s^{n}\right)}-H(S)\right| \leq \gamma_{n}\right\} .
$$

Then, it is well-known that (e.g., see [23, Theorem 3.1.2]) $\mathcal{T}_{\gamma_{n}}$ satisfies the following properties:

$$
\begin{aligned}
& \lim _{n \rightarrow \infty} \operatorname{Pr}\left\{S^{n} \in \mathcal{T}_{\gamma_{n}}\right\}=1 \\
& \left|\mathcal{T}_{\gamma_{n}}\right| \leq 2^{n\left\{H(S)+\gamma_{n}\right\}} \text { for all } n \geq 1 .
\end{aligned}
$$

For an arbitrary $\ell \geq 0$, let $\mathcal{L}_{n} \stackrel{\text { def }}{=}\left\{0,1, \ldots, L_{n}-1\right\}$ and $\mathcal{M}_{n} \stackrel{\text { def }}{=}$ $\left\{0,1, \ldots, M_{n}-1\right\}$ be sets of integers where $L_{n} \stackrel{\text { def }}{=}\left\lfloor 2^{n \ell}\right\rfloor$ and $M_{n} \stackrel{\text { def }}{=}\left|\mathcal{T}_{\gamma_{n}}\right|$.

In the following, we construct a sequence $\left\{\left(\varphi_{n}^{*}, \psi_{n}^{*}\right)\right\}_{n=1}^{\infty}$ of an encoder $\varphi_{n}^{*}$ and a decoder $\psi_{n}^{*}$ that asymptotically realizes a $(2,2)$-threshold scheme with correlation level $\ell$ satisfying $\left|\mathcal{X}_{n}\right|=\left|\mathcal{Y}_{n}\right|=\left|\mathcal{U}_{n}\right|=L_{n}\left(M_{n}+1\right)$.

The encoder $\varphi_{n}^{*}$ can be constructed as follows: Since $M_{n}=$ $\left|\mathcal{T}_{\gamma_{n}}\right|$, there exists a bijection $\xi_{n}: \mathcal{T}_{\gamma_{n}} \rightarrow \mathcal{M}_{n}$. Furthermore, define a map $\xi_{n}^{+}: \mathcal{S}^{n} \rightarrow \mathcal{M}_{n}^{+}$where $\mathcal{M}_{n}^{+} \stackrel{\text { def }}{=} \mathcal{M}_{n} \cup\left\{M_{n}\right\}$ by

$$
\xi_{n}^{+}\left(s^{n}\right)=\left\{\begin{array}{cl}
\xi_{n}\left(s^{n}\right), & \text { if } s^{n} \in \mathcal{T}_{\gamma_{n}} \\
M_{n}, & \text { otherwise }
\end{array}\right.
$$

and let $Z_{n} \stackrel{\text { def }}{=} \xi_{n}^{+}\left(S^{n}\right)$. Denote by $U_{n}^{\mathcal{L}}$ and $U_{n}^{\mathcal{M}}$ the random variables subject to the uniform distribution on $\mathcal{L}_{n}$ and $\mathcal{M}_{n}^{+}$, respectively, and define $U_{n}=\left(U_{n}^{\mathcal{L}}, U_{n}^{\mathcal{M}}\right)$. In addition, we define two shares by

$$
\begin{aligned}
X_{n} & =\left(X_{n}^{\mathcal{L}}, X_{n}^{\mathcal{M}}\right)=\left(U_{n}^{\mathcal{L}}, Z_{n} \ominus U_{n}^{\mathcal{M}}\right) \in \mathcal{L}_{n} \times \mathcal{M}_{n}^{+} \\
Y_{n} & =\left(U_{n}^{\mathcal{L}}, U_{n}^{\mathcal{M}}\right) \in \mathcal{L}_{n} \times \mathcal{M}_{n}^{+}
\end{aligned}
$$

where $\ominus$ represents the subtraction of modulo $M_{n}+1$. 
Next, let us define the decoder $\psi_{n}^{*}$. Let $x_{n}=\left(x_{n}^{\mathcal{L}}, x_{n}^{\mathcal{M}}\right) \in$ $\mathcal{L}_{n} \times \mathcal{M}_{n}^{+}$and $y_{n}=\left(y_{n}^{\mathcal{L}}, y_{n}^{\mathcal{M}}\right) \in \mathcal{L}_{n} \times \mathcal{M}_{n}^{+}$be the inputs to the decoder. Then, the decoder $\psi_{n}^{*}$ first checks whether $x_{n}^{\mathcal{L}}=y_{n}^{\mathcal{L}}$ holds or not. If $x_{n}^{\mathcal{L}} \neq y_{n}^{\mathcal{L}}$, the decoder judges that impersonation attack has occurred and outputs $\perp$. On the other hand, if $x_{n}^{\mathcal{L}}=y_{n}^{\mathcal{L}}$, the decoder computes $x_{n}^{\mathcal{M}} \oplus y_{n}^{\mathcal{M}}$, where $\oplus$ denotes the addition of modulo $M_{n}+1$. If $x_{n}^{\mathcal{M}} \oplus y_{n}^{\mathcal{M}}=M_{n}$, the decoder outputs $\perp$ since the decoding error occurs in such a case. Otherwise, the decoder outputs $\xi_{n}^{-1}\left(x_{n}^{\mathcal{M}} \oplus y_{n}^{\mathcal{M}}\right)$ where $\xi_{n}^{-1}: \mathcal{M}_{n} \rightarrow \mathcal{T}_{\gamma_{n}}$ is the inverse map of $\xi_{n}$. Summarizing, the decoder $\psi_{n}^{*}$ is written as

$$
\begin{aligned}
& \psi_{n}^{*}\left(x_{n}, y_{n}\right) \\
& \quad=\left\{\begin{array}{cl}
\xi_{n}^{-1}\left(x_{n}^{\mathcal{M}} \oplus y_{n}^{\mathcal{M}}\right), & \text { if } x_{n}^{\mathcal{L}}=y_{n}^{\mathcal{L}} \text { and } \\
& x_{n}^{\mathcal{M}} \oplus y_{n}^{\mathcal{M}} \neq M_{n} \text { are satisfied } \\
\perp, & \text { otherwise }
\end{array}\right.
\end{aligned}
$$

and the acceptance region of $\psi_{n}^{*}$ is given by

$$
\begin{aligned}
& \mathcal{A}_{n}=\left\{\left(x_{n}, y_{n}\right) \in \mathcal{X}_{n} \times \mathcal{Y}_{n}: x_{n}^{\mathcal{L}}=y_{n}^{\mathcal{L}}\right. \\
&\text { and } \left.x_{n}^{\mathcal{M}} \oplus y_{n}^{\mathcal{M}} \in \mathcal{M}_{n}\right\} .
\end{aligned}
$$

Hereafter, we prove that the above sequence $\left\{\left(\varphi_{n}^{*}, \psi_{n}^{*}\right)\right\}_{n=1}^{\infty}$ realizes the optimal $(2,2)$-threshold scheme with correlation level $\ell$ that asymptotically attains all the bounds in ([17) - ([20). It suffices to prove Claims $\square-\$$ below.

Claim 1: For an arbitrarily small $\gamma>0$, the rates of $X_{n}$, $Y_{n}$ and $U_{n}$ can be less than $H(S)+\ell+\gamma$ for all sufficiently

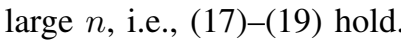

Claim 2: The limit inferior of the minimum exponent in the success probabilities of impersonation attacks is at least $\ell$, i.e., (20I) holds.

Claim 3: The decoding error probability for the legitimate shares vanishes as $n$ goes to infinity, i.e., (पD) holds.

Claim 4: For all $n \geq 1$, the $n$ source outputs $S^{n}$ are secure against the leakage from one of $X_{n}$ and $Y_{n}$, i.e., (2II) holds.

Claim 5: The correlation level between $X_{n}$ and $Y_{n}$ equals to $\ell$, i.e., (12) holds.

Proof of Claim प: In order to evaluate the share rates and the randomness given by ([1])-([प), observe that

$$
\begin{aligned}
\log \left|\mathcal{X}_{n}\right| & =\log \left|\mathcal{Y}_{n}\right|=\log \left|\mathcal{U}_{n}\right| \\
& =\log \left\{L_{n}\left(M_{n}+1\right)\right\} \\
& =\log \left\{\left\lfloor 2^{n \ell}\right\rfloor\left(\left|\mathcal{T}_{\gamma_{n}}\right|+1\right)\right\} \\
& \leq n\left\{H(S)+\ell+\gamma_{n}\right\}+1
\end{aligned}
$$

where the last inequality follows from (4II). Hence, it holds that

$\frac{1}{n} \log \left|\mathcal{X}_{n}\right|=\frac{1}{n} \log \left|\mathcal{Y}_{n}\right|=\frac{1}{n} \log \left|\mathcal{U}_{n}\right| \leq H(S)+\ell+\gamma_{n}+\frac{1}{n}$.

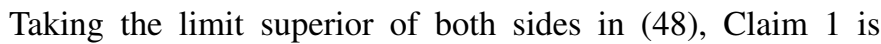
established.
Proof of Claim घ: We evaluate $P_{n}^{X}$ in the following way:

$$
\begin{aligned}
P_{n}^{X} & =\max _{P_{\bar{X}_{n}}} \operatorname{Pr}\left\{\left(\bar{X}_{n}, Y_{n}\right) \in \mathcal{A}_{n}\right\} \\
& =\max _{P_{\bar{X}_{n}}} \operatorname{Pr}\left\{\bar{X}_{n}^{\mathcal{L}}=Y_{n}^{\mathcal{L}} \text { and } \bar{X}_{n}^{\mathcal{M}} \oplus Y_{n}^{\mathcal{M}} \in \mathcal{T}_{\gamma_{n}}\right\} \\
& \leq \max _{P_{\bar{X}_{n}}} \operatorname{Pr}\left\{\bar{X}_{n}^{\mathcal{L}}=Y_{n}^{\mathcal{L}}\right\}=\max _{P_{\bar{X}_{n}^{\mathcal{L}}}} \operatorname{Pr}\left\{\bar{X}_{n}^{\mathcal{L}}=U_{n}^{\mathcal{L}}\right\} \\
& \stackrel{(\text { a) }}{=} \max _{P_{\bar{X}_{n}^{\mathcal{L}}}} \sum_{x_{n}^{\mathcal{L}} \in \mathcal{L}_{n}} P_{\bar{X}_{n}^{\mathcal{L}}}\left(x_{n}^{\mathcal{L}}\right) P_{U_{n}^{\mathcal{L}}}\left(x_{n}^{\mathcal{L}}\right) \\
& \stackrel{(\mathrm{b})}{=} \frac{1}{L_{n}} \max _{P_{\bar{X}_{n}^{\mathcal{L}}}} \sum_{x_{n}^{\mathcal{L}} \in \mathcal{L}_{n}} P_{\bar{X}_{n}^{\mathcal{L}}}\left(x_{n}^{\mathcal{L}}\right)=\frac{1}{L_{n}}
\end{aligned}
$$

where $\bar{X}_{n} \stackrel{\text { def }}{=}\left(\bar{X}_{n}^{\mathcal{L}}, \bar{X}_{n}^{\mathcal{M}}\right) \in \mathcal{L}_{n} \times \mathcal{M}_{n}^{+}$and $Y_{n} \stackrel{\text { def }}{=}$ $\left(Y_{n}^{\mathcal{L}}, Y_{n}^{\mathcal{M}}\right)=\left(U_{n}^{\mathcal{L}}, U_{n}^{\mathcal{M}}\right) \in \mathcal{L}_{n} \times \mathcal{M}_{n}^{+}$, and the marked equalities follow from the following reasons:

(a) $\bar{X}_{n}^{\mathcal{L}}$ and $U_{n}^{\mathcal{L}}$ are independent.

(b) $P_{U_{n}^{\mathcal{L}}}\left(x_{n}^{\mathcal{L}}\right)=1 / L_{n}$ holds for all $x_{n}^{\mathcal{L}} \in \mathcal{L}_{n}$.

Similarly, noticing the fact that $X_{n}^{\mathcal{L}}=U_{n}^{\mathcal{L}}$, we also have $P_{n}^{Y} \leq$ $1 / L_{n}$, and therefore, we conclude that

$$
\begin{array}{r}
\liminf _{n \rightarrow \infty} \min \{- \\
\left.\geq \frac{1}{n} \log P_{n}^{X},-\frac{1}{n} \log P_{n}^{Y}\right\} \\
\geq \liminf _{n \rightarrow \infty} \frac{1}{n} \log L_{n}=\ell .
\end{array}
$$

Proof of Claim [1: Since every legitimate pair $\left(x_{n}, y_{n}\right) \in \mathcal{A}_{n}$ of shares is decoded by $\varphi_{n}^{*}$ without error, the decoding error happens only if the decoder $\psi_{n}^{*}$ outputs $\perp$ for a pair of legitimate shares $\left(x_{n}, y_{n}\right)$. Hence, the decoding error probability $P_{n}^{e}$ can be written as

$$
\begin{aligned}
P_{n}^{e} & =\operatorname{Pr}\left\{\psi_{n}^{*}\left(X_{n}, Y_{n}\right)=\perp\right\} \\
& =\operatorname{Pr}\left\{\xi_{n}^{+}\left(S^{n}\right)=M_{n}\right\} \\
& =\operatorname{Pr}\left\{S^{n} \notin \mathcal{T}_{\gamma_{n}}\right\} .
\end{aligned}
$$

Therefore, it follows from (401) that $\lim _{n \rightarrow \infty} P_{n}^{e}=1-$ $\lim _{n \rightarrow \infty} \operatorname{Pr}\left\{S^{n} \in \mathcal{T}_{\gamma_{n}}\right\}=0$.

Proof of Claim 国: First, we note that $Z_{n}$ and $X_{n}^{\mathcal{M}}=Z_{n} \ominus$ $U_{n}^{\mathcal{M}}$ are independent because of non-negativity of the mutual information and

$$
\begin{aligned}
I\left(Z_{n} ; Z_{n} \ominus U_{n}^{\mathcal{M}}\right)= & H\left(Z_{n}\right)+H\left(Z_{n} \ominus U_{n}^{\mathcal{M}}\right) \\
& -H\left(Z_{n}, Z_{n} \ominus U_{n}^{\mathcal{M}}\right) \\
= & H\left(Z_{n}\right)+H\left(Z_{n} \ominus U_{n}^{\mathcal{M}}\right)-H\left(Z_{n}, U_{n}^{\mathcal{M}}\right) \\
= & H\left(Z_{n} \ominus U_{n}^{\mathcal{M}}\right)-H\left(U_{n}^{\mathcal{M}}\right) \\
\leq & 0
\end{aligned}
$$

where the last inequality holds because $Z_{n} \ominus U_{n}^{\mathcal{M}} \in \mathcal{M}_{n}^{+}$and $U_{n}^{\mathcal{M}}$ is subject to the uniform distribution on $\mathcal{M}_{n}^{+}$. Hence, $Z_{n}$ and $X_{n}=\left(X_{n}^{\mathcal{L}}, X_{n}^{\mathcal{M}}\right)$ are also independent because

$$
\begin{aligned}
I\left(Z_{n} ; X_{n}\right) & =I\left(Z_{n} ; X_{n}^{\mathcal{L}} X_{n}^{\mathcal{M}}\right) \\
& =I\left(Z_{n} ; X_{n}^{\mathcal{M}}\right)+I\left(Z_{n} ; X_{n}^{\mathcal{L}} \mid X_{n}^{\mathcal{M}}\right) \\
& =0
\end{aligned}
$$


where the last equality follows since $I\left(Z_{n} ; X_{n}^{\mathcal{M}}\right)=0$, and $Z_{n}, X_{n}^{\mathcal{M}}$, and $X_{n}^{\mathcal{L}}$ form a Markov chain in this order.

In order to show (2II), it is sufficient to prove that $I\left(S^{n} ; X_{n}\right)=0$ for all $n \geq 1$ because $I\left(S^{n} ; Y_{n}\right)=0$ for any $n \geq 1$ trivially holds from the fact that $S^{n}$ and $Y_{n}=\left(U_{n}^{\mathcal{L}}, U_{n}^{\mathcal{M}}\right)$ are independent. In addition, $I\left(S_{n} ; X_{n}\right)=0$ is established from $I\left(S^{n} ; X_{n}\right) \leq I\left(Z_{n} ; X_{n}\right)=0$ which is obtained by the information processing inequality [23, Theorem 2.8.1] for a Markov chain $S_{n} \rightarrow Z_{n} \rightarrow X_{n}$, and recalling (52).

Proof of Claim [1]: The correlation level can be evaluated as follows. Note that the mutual information of shares $X_{n}$ and $Y_{n}$ satisfies

$$
\begin{aligned}
& I\left(X_{n} ; Y_{n}\right)=I\left(X_{n}^{\mathcal{L}} X_{n}^{\mathcal{M}} ; Y_{n}^{\mathcal{L}} Y_{n}^{\mathcal{M}}\right) \\
& =I\left(U_{n}^{\mathcal{L}} X_{n}^{\mathcal{M}} ; U_{n}^{\mathcal{L}} U_{n}^{\mathcal{M}}\right) \\
& =H\left(U_{n}^{\mathcal{L}} X_{n}^{\mathcal{M}}\right)-H\left(X_{n}^{\mathcal{M}} \mid U_{n}^{\mathcal{L}} U_{n}^{\mathcal{M}}\right) \\
& \text { - } H\left(U_{n}^{\mathcal{L}} \mid X_{n}^{\mathcal{M}} U_{n}^{\mathcal{L}} U_{n}^{\mathcal{M}}\right) \\
& \stackrel{\text { (c) }}{=} H\left(U_{n}^{\mathcal{L}}\right)+H\left(X_{n}^{\mathcal{M}}\right)-H\left(X_{n}^{\mathcal{M}} \mid U_{n}^{\mathcal{M}}\right) \\
& \stackrel{(\mathrm{d})}{=} H\left(U_{n}^{\mathcal{L}}\right)+H\left(X_{n}^{\mathcal{M}}\right)-H\left(Z_{n}\right)
\end{aligned}
$$

where the marked equalities hold because of the following reasons:

(c) $U_{n}^{\mathcal{L}}$ and $X_{n}^{\mathcal{M}}$ are independent, and $U_{n}^{\mathcal{L}}, U_{n}^{\mathcal{M}}$, and $X_{n}^{\mathcal{M}}$ form a Markov chain in this order.

(d) It follows that $H\left(X_{n}^{\mathcal{M}} \mid U_{n}^{\mathcal{M}}\right)=H\left(Z_{n} \ominus U_{n}^{\mathcal{M}} \mid U_{n}^{\mathcal{M}}\right)=$ $H\left(Z_{n} \mid U_{n}^{\mathcal{M}}\right)=H\left(Z_{n}\right)$ due to the independence of $S^{n}$ and $U_{n}$.

Hereafter, we evaluate the terms on the right hand side of (53). It is easy to see that

$$
H\left(U_{n}^{\mathcal{L}}\right)=\log L_{n}=\log \left\lfloor 2^{n \ell}\right\rfloor .
$$

The second term in the right hand side of (53) can be evaluated as

$$
\begin{aligned}
H\left(X_{n}^{\mathcal{M}}\right) & \leq \log \left(M_{n}+1\right) \\
& =\log \left(\left|\mathcal{T}_{\gamma_{n}}\right|+1\right) \\
& \leq n\left\{H(S)+\gamma_{n}\right\}+1
\end{aligned}
$$

where the last inequality follows from (4II). In order to evaluate the last term on the right hand side of (53), we set $\delta_{n}=$ $\operatorname{Pr}\left\{\xi_{n}^{+}\left(S^{n}\right)=M_{n}\right\}=\operatorname{Pr}\left\{S^{n} \notin \mathcal{T}_{\gamma_{n}}\right\}$. Clearly, $\lim _{n \rightarrow \infty} \delta_{n}=$ 0 from (40). Since the map $\xi_{n}: \mathcal{T}_{\gamma_{n}} \rightarrow \mathcal{M}_{n}$ is bijective, we have

$$
\begin{aligned}
H\left(Z_{n}\right) & =H\left(\xi_{n}^{+}\left(S^{n}\right)\right) \\
& =\sum_{s^{n} \in \mathcal{T}_{\gamma_{n}}} P_{S^{n}}\left(s^{n}\right) \log \frac{1}{P_{S^{n}}\left(s^{n}\right)}+\delta_{n} \log \frac{1}{\delta_{n}} \\
& \geq \sum_{s^{n} \in \mathcal{T}_{\gamma_{n}}} P_{S^{n}}\left(s^{n}\right) n\left\{H(S)-\gamma_{n}\right\}-\delta_{n} \log \delta_{n} \\
& =\left(1-\delta_{n}\right) n\left\{H(S)-\gamma_{n}\right\}-\delta_{n} \log \delta_{n}
\end{aligned}
$$

where the inequality holds because of (41). Hence, we have from (55) and (56) that

$$
\begin{aligned}
H\left(X_{n}^{\mathcal{M}}\right) & -H\left(Z_{n}\right) \\
& \leq n \delta_{n} H(S)+n\left(2-\delta_{n}\right) \gamma_{n}+\delta_{n} \log \delta_{n}+1 .
\end{aligned}
$$

On the other hand, it is easy to see with the same reason for the equality (d) in (53) that

$$
H\left(X_{n}^{\mathcal{M}}\right)-H\left(Z_{n}\right) \geq H\left(X_{n}^{\mathcal{M}} \mid U_{n}^{\mathcal{M}}\right)-H\left(Z_{n}\right)=0 .
$$

Summarizing, we have from (53)), (54), (57)), and (58) that

$$
\begin{aligned}
\frac{1}{n} \log \left\lfloor 2^{n \ell}\right\rfloor \leq & \frac{1}{n} I\left(X_{n} ; Y_{n}\right) \\
\leq & \frac{1}{n} \log \left\lfloor 2^{n \ell}\right\rfloor+\delta_{n} H(S) \\
& +\left(2-\delta_{n}\right) \gamma_{n}+\frac{1}{n}\left(\delta_{n} \log \delta_{n}+1\right) .
\end{aligned}
$$

By taking the limit of both sides of (59) and noticing that $\lim _{n \rightarrow \infty} \gamma_{n}=\lim _{n \rightarrow \infty} \delta_{n}=0$, we have

$$
\lim _{n \rightarrow \infty} \frac{1}{n} I\left(X_{n} ; Y_{n}\right)=\ell
$$

Since Claims $\square-\square$ are verified, Theorem $\square$ is proved.

Remark 4: The claim of Theorem $\square$ is valid for the class of stationary ergodic sources if the entropy $H(S)$ in Theorem Q is replaced with the entropy rate $H \stackrel{\text { def }}{=} \lim _{n \rightarrow \infty} H\left(S^{n}\right) / n$. This fact is obtained by a slight modification of the proof of Theorem $\square$ followed by the diagonal line argument [25, Theorem 1.8.2]. First, by the asymptotic equipartition property [23, Theorem 3.1.2], we have

$$
\lim _{n \rightarrow \infty} \operatorname{Pr}\left\{S^{n} \in \mathcal{T}_{n, \gamma}\right\}=1
$$

for any constant $\gamma>0$, where

$$
\mathcal{T}_{n, \gamma} \stackrel{\text { def }}{=}\left\{s^{n} \in \mathcal{S}^{n}:\left|\frac{1}{n} \log \frac{1}{P_{S^{n}}\left(s^{n}\right)}-H\right| \leq \gamma\right\}
$$

and $H$ denotes the entropy rate of the source. We construct an encoder $\varphi_{n, \gamma}^{*}$ and a decoder $\psi_{n, \gamma}^{*}$ in the same way as in the proof of Theorem [2. It is easily checked that $\left\{\left(\varphi_{n, \gamma}^{*}, \psi_{n, \gamma}^{*}\right)\right\}_{n=1}^{\infty}$ asymptotically realizes the $(2,2)$-threshold scheme. In addition, by the same argument with (48) and (59), $\left\{\left(\varphi_{n, \gamma}^{*}, \psi_{n, \gamma}^{*}\right)\right\}_{n=1}^{\infty}$ satisfies

$$
\frac{1}{n} \log \left|\mathcal{X}_{n}\right|=\frac{1}{n} \log \left|\mathcal{Y}_{n}\right|=\frac{1}{n} \log \left|\mathcal{U}_{n}\right| \leq H+\ell+\gamma+\frac{1}{n}
$$

and

$$
\begin{aligned}
\frac{1}{n} \log \left\lfloor 2^{n \ell}\right\rfloor \leq & \frac{1}{n} I\left(X_{n} ; Y_{n}\right) \\
\leq & \frac{1}{n} \log \left\lfloor 2^{n \ell}\right\rfloor+\delta_{n, \gamma} H+\left(2-\delta_{n, \gamma}\right) \gamma \\
& +\frac{1}{n}\left(\delta_{n, \gamma} \log \delta_{n, \gamma}+1\right)
\end{aligned}
$$

where $\delta_{n, \gamma} \stackrel{\text { def }}{=} \operatorname{Pr}\left\{S^{n} \notin \mathcal{T}_{n, \gamma}\right\} \rightarrow 0$ as $n \rightarrow \infty$. Note that (64) implies that

$$
\left|\frac{1}{n} I\left(X_{n} ; Y_{n}\right)-\ell\right| \leq 3 \gamma \text { for all } n \geq N_{0}(\gamma) .
$$

We now fix a sequence $\left\{\gamma_{m}\right\}_{m=1}^{\infty}$ satisfying $\gamma_{0}>\gamma_{1}>$ $\cdots>\gamma_{m}>\cdots>0$ arbitrarily and define $N_{0}=1$ and $N_{m}, m=1,2, \ldots$, as the minimum integer $N$ satisfying $\left|I\left(X_{n} ; Y_{n}\right) / n-\ell\right| \leq 3 \gamma_{m}$ for all $n \geq N$. Obviously, 
$\left\{N_{m}\right\}_{m=1}^{\infty}$ is monotone nondecreasing. We define $\left(\varphi_{n}^{*}, \psi_{n}^{*}\right)$ as $\left(\varphi_{n, \gamma_{0}}^{*}, \psi_{n, \gamma_{0}}^{*}\right)$ for each $1 \leq n<N_{1}$ and $\left(\varphi_{n, \gamma_{m}}^{*}, \psi_{n, \gamma_{m}}^{*}\right)$ for each $N_{m} \leq n<N_{m+1}, m=1,2, \ldots$. Then, in view of (63), (65) and $\gamma_{m} \downarrow 0$ as $m \rightarrow \infty$, we can conclude that $\left\{\left(\varphi_{n}^{*}, \psi_{n}^{*}\right)\right\}_{n=1}^{\infty}$ satisfies

$$
\begin{aligned}
\limsup _{n \rightarrow \infty} \frac{1}{n} \log \left|\mathcal{X}_{n}\right|=\limsup _{n \rightarrow \infty} \frac{1}{n} \log \left|\mathcal{Y}_{n}\right| & =\limsup _{n \rightarrow \infty} \frac{1}{n} \log \left|\mathcal{U}_{n}\right| \\
& \leq H+\ell
\end{aligned}
$$

and

$$
\lim _{n \rightarrow \infty} \frac{1}{n} I\left(X_{n} ; Y_{n}\right)=\ell
$$

\section{Another Optimal Scheme Using Symbolwise ENCODING}

In Section WI, we have shown by using blockwise coding that the sequence $\left\{\left(\varphi_{n}^{*}, \psi_{n}^{*}\right)\right\}_{n=1}^{\infty}$ of an encoder and a decoder realizes the asymptotically optimal $(2,2)$-threshold scheme with correlation level $\ell$. In addition, $\left\{\left(\varphi_{n}^{*}, \psi_{n}^{*}\right)\right\}_{n=1}^{\infty}$ also attains the maximum exponent in the success probabilities of impersonation attack which is given by $\ell$. In this section, by using a symbolwise encoding, we give a simple construction of $\left\{\left(\varphi_{n}^{*}, \psi_{n}^{*}\right)\right\}_{n=1}^{\infty}$ that realizes the asymptotically optimal $(2,2)$ threshold scheme with correlation level $\ell$ and the exponent in the success probability of impersonation attacks equals to $\ell$. In this construction, we use a pair $(f, g)$ of an encoder $f$ and a decoder $g$ for a $(2,2)$-threshold scheme for a single source output $S$. In addition, a one-sided test is used to detect the impersonation attacks.

Let $S, U, X$ and $Y$ be random variables of a secret, a random number, and two shares taking values in finite sets $\mathcal{S}, \mathcal{U}, \mathcal{X}$ and $\mathcal{Y}$ respectively. For a non-negative number $\ell$, we first define a pair $(f, g)$ of an encoder $f$ and a decoder $g$ for a $(2,2)$ threshold scheme with correlation level $\ell$. That is, the encoder $f: \mathcal{S} \times \mathcal{U} \rightarrow \mathcal{X} \times \mathcal{Y}$ is defined to be a deterministic map satisfying

$$
\begin{aligned}
H(S \mid X) & =H(S \mid Y)=H(S) \\
H(S \mid X Y) & =0
\end{aligned}
$$

in addition to

$$
I(X ; Y)=\ell
$$

where shares $X$ and $Y$ are determined by $(X, Y)=f(S, U)$. Note that (68) and (69) are the ordinary requirements for $(2,2)$-threshold schemes, i.e., (68) guarantees that any information of $S$ does not leak from either one of the shares, and (69) implies that the secret $S$ can be decoded from $X$ and $Y$ without error. Hence, let $g: \mathcal{X} \times \mathcal{Y} \rightarrow \mathcal{S} \cup\{\lambda\}$ be a decoder corresponding to $f$ and satisfying $g(x, y)=\lambda$ for every $(x, y) \in \mathcal{X} \times \mathcal{Y}$ that does not belong to the range of $f$. Furthermore, (ZD) means that the correlation level of $X$ and $Y$ generated by the encoder $f$ is equal to $\ell$. We say that a pair $(f, g)$ of an encoder $f$ and a decoder $g$ realizes a $(2,2)$-threshold scheme with correlation level $\ell$ in the nonasymptotic sense if $(f, g)$ satisfies (68)-([0)). In addition, it is shown in [26] that

$$
\min \{|\mathcal{X}|,|\mathcal{Y}|,|\mathcal{U}|\} \geq|\mathcal{S}|
$$

must be satisfied for any encoder of $(2,2)$-threshold schemes satisfying (68) and (69). Hence, we also impose ([U]) on $f$ in addition to (68) -(701).

In this setting, we define an encoder $\varphi_{n}^{*}: \mathcal{S}^{n} \times \mathcal{U}^{n} \rightarrow$ $\mathcal{X}^{n} \times \mathcal{Y}^{n}$ as the repeated application of $f: \mathcal{S} \times \mathcal{U} \rightarrow \mathcal{X} \times \mathcal{Y}$ to $\left(S_{i}, U_{i}\right), i=1,2, \ldots, n$, which can be written as

$$
\varphi_{n}^{*}\left(s^{n}, u^{n}\right) \stackrel{\text { def }}{=} f\left(s_{1}, u_{1}\right) f\left(s_{2}, u_{2}\right) \cdots f\left(s_{n}, u_{n}\right)
$$

where $s^{n} \stackrel{\text { def }}{=} s_{1} s_{2} \cdots s_{n} \in \mathcal{S}^{n}$ and $u^{n} \stackrel{\text { def }}{=} u_{1} u_{2} \cdots u_{n} \in \mathcal{U}^{n}$ are $n$ secrets and $n$ random numbers, respectively. Hence, the two shares $X^{n}=X_{1} X_{2} \cdots X_{n} \in \mathcal{X}^{n}$ and $Y^{n}=Y_{1} Y_{2} \cdots Y_{n} \in$ $\mathcal{Y}^{n}$ are i.i.d. copies of $X$ and $Y$, respectively, where $\left(X_{i}, Y_{i}\right)=$ $f\left(S_{i}, U_{i}\right)$.

Furthermore, we define

$$
\begin{aligned}
\mathcal{A}_{n}^{*}=\{ & \left(x^{n}, y^{n}\right) \in \mathcal{X}^{n} \times \mathcal{Y}^{n}: \\
& \left.\frac{1}{n} \log \frac{P_{X Y}\left(x^{n}, y^{n}\right)}{P_{X^{n}}\left(x^{n}\right) P_{Y^{n}}\left(y^{n}\right)}>I(X ; Y)-\gamma_{n}\right\}
\end{aligned}
$$

where $\gamma_{n}$ is an arbitrary sequence of positive integers $\left\{\gamma_{n}\right\}_{n=1}^{\infty}$ satisfying $\lim _{n \rightarrow \infty} \gamma_{n}=0$ and $\lim _{n \rightarrow \infty} \sqrt{n} \gamma_{n}=\infty$. Then, legitimate shares belong to $\mathcal{A}_{n}^{*}$ with high probability if $n$ is sufficiently large since

$$
\lim _{n \rightarrow \infty} \operatorname{Pr}\left\{\left(X^{n}, Y^{n}\right) \in \mathcal{A}_{n}^{*}\right\}=1
$$

holds from the law of large numbers. Hence, we regard the received shares as legitimate if they belong to $\mathcal{A}_{n}^{*}$, and decode them by the decoder $g_{n}$ corresponding to the encoder $\varphi_{n}^{*}$ in (72), where $g_{n}$ can be written as

$$
g_{n}\left(x^{n}, y^{n}\right) \stackrel{\text { def }}{=} g\left(x_{1}, y_{1}\right) g\left(x_{2}, y_{2}\right) \cdots g\left(x_{n}, y_{n}\right) .
$$

In addition, the decoder $\psi_{n}^{*}: \mathcal{X}^{n} \times \mathcal{Y}^{n} \rightarrow \mathcal{S}^{n} \cup\{\perp\}$ is defined by

$$
\psi_{n}^{*}\left(x^{n}, y^{n}\right)=\left\{\begin{array}{cl}
g_{n}\left(x^{n}, y^{n}\right), & \text { if }\left(x^{n}, y^{n}\right) \in \mathcal{A}_{n}^{*} \\
\perp, & \text { otherwise }
\end{array}\right.
$$

where $\perp$ means that the impersonation attack, i.e., (a1) or (a2) in Section III, is detected.

According to ([4), every $\left(x^{n}, y^{n}\right) \in \mathcal{A}_{n}^{*}$ satisfies $P_{X^{n} Y^{n}}\left(x^{n}, y^{n}\right)>0$, which is equivalent to $P_{X Y}\left(x_{i}, y_{i}\right)>0$, i.e., $g\left(x_{i}, y_{i}\right) \neq \lambda$, for all $i=1,2, \ldots, n$. Hence, for every $\left(x^{n}, y^{n}\right) \in \mathcal{A}_{n}^{*}$, there uniquely exists $s^{n} \in \mathcal{S}^{n}$ that satisfies $g_{n}\left(x^{n}, y^{n}\right)=s^{n}$ whether the received shares $x^{n}$ and $y^{n}$ are legitimate or not. Furthermore, if the pair of shares $\left(x^{n}, y^{n}\right) \in \mathcal{A}_{n}^{*}$ is legitimate, the secret is reproduced without error due to the definitions of $f$ and $\varphi_{n}^{*}$. More precisely, $\psi_{n}^{*}\left(x^{n}, y^{n}\right)=g_{n}\left(x^{n}, y^{n}\right)=s^{n}$ holds for every $u^{n} \in \mathcal{U}^{n}, s^{n} \in \mathcal{S}^{n}, x^{n} \in \mathcal{X}^{n}$ and $y^{n} \in \mathcal{Y}^{n}$ satisfying $\varphi_{n}^{*}\left(s^{n}, u^{n}\right)=\left(x^{n}, y^{n}\right) \in \mathcal{A}_{n}^{*}$.

The above sequence $\left\{\left(\varphi_{n}^{*}, \psi_{n}^{*}\right)\right\}_{n=1}^{\infty}$ defined by (II2) and (106) realizes an asymptotic $(2,2)$-threshold scheme with correlation level $\ell$. 
Theorem 3: Let $(f, g)$ be any pair of an encoder and a decoder that realizes a $(2,2)$-threshold scheme with correlation level $\ell$ in the non-asymptotic sense. Then, the sequence $\left\{\left(\varphi_{n}^{*}, \psi_{n}^{*}\right)\right\}_{n=1}^{\infty}$ defined by (122) and (106) satisfies for all $n \geq 1$ that

$$
\begin{aligned}
P_{n}^{e} & =\operatorname{Pr}\left\{\left(X^{n}, Y^{n}\right) \notin \mathcal{A}_{n}^{*}\right\} \\
H\left(S^{n} \mid X^{n}\right) & =H\left(S^{n} \mid Y^{n}\right)=H\left(S^{n}\right) \\
I\left(X^{n} ; Y^{n}\right) & =n \ell
\end{aligned}
$$

which obviously realizes an asymptotic (2,2)-threshold scheme with correlation level $\ell$. In addition, this $\left\{\left(\varphi_{n}^{*}, \psi_{n}^{*}\right)\right\}_{n=1}^{\infty}$ satisfies (ㅁ)).

Proof of Theorem [1: First, we prove (पDI). If there is a oneto-one correspondence between $\left(s^{n}, u^{n}\right)$ and $\left(x^{n}, y^{n}\right)$, ([77) is obvious. We show that (IZ) holds for any pair of $f$ and $g$ satisfying (68) and (69) which does not guarantee the existence of such a one-to-one correspondence.

Define

$$
\begin{array}{r}
\mathcal{D}_{n}^{*}\left(x^{n}, y^{n}\right)=\left\{\left(s^{n}, u^{n}\right): \varphi_{n}^{*}\left(s^{n}, u^{n}\right)=\left(x^{n}, y^{n}\right)\right. \\
\text { and } \left.\psi_{n}^{*}\left(x^{n}, y^{n}\right)=s^{n}\right\} .
\end{array}
$$

Recalling that $\psi_{n}^{*}\left(x^{n}, y^{n}\right)=g_{n}\left(x^{n}, y^{n}\right)=s^{n}$ for every $u^{n} \in$ $\mathcal{U}^{n}, s^{n} \in \mathcal{S}^{n}, x^{n} \in \mathcal{X}^{n}$ and $y^{n} \in \mathcal{Y}^{n}$ satisfying $\varphi_{n}^{*}\left(s^{n}, u^{n}\right)=$ $\left(x^{n}, y^{n}\right) \in \mathcal{A}_{n}^{*}$, it holds for all $\left(x^{n}, y^{n}\right) \in \mathcal{A}_{n}^{*}$ that

$$
\begin{aligned}
\mathcal{D}_{n}\left(x^{n}, y^{n}\right)= & \left\{\left(s^{n}, u^{n}\right): \varphi_{n}^{*}\left(s^{n}, u^{n}\right)=\left(x^{n}, y^{n}\right)\right. \\
& \text { and } \left.g_{n}\left(x^{n}, y^{n}\right)=s^{n}\right\} \\
= & \left\{\left(s^{n}, u^{n}\right): \varphi_{n}^{*}\left(s^{n}, u^{n}\right)=\left(x^{n}, y^{n}\right)\right\} \\
& \stackrel{\text { def }}{=} \varphi_{n}^{*-1}\left(x^{n}, y^{n}\right)
\end{aligned}
$$

where $\varphi_{n}^{*-1}\left(x^{n}, y^{n}\right)$ means the inverse image of $\left(x^{n}, y^{n}\right)$.

Next, we define

$$
\mathcal{D}_{n}=\left\{\left(s^{n}, u^{n}\right): \psi_{n}^{*}\left(\varphi_{n}^{*}\left(s^{n}, u^{n}\right)\right)=s^{n}\right\} .
$$

Then, since $\psi_{n}^{*}\left(x^{n}, y^{n}\right)=\perp$ for all $\left(x^{n}, y^{n}\right) \notin \mathcal{A}_{n}^{*}$ and $s^{n}$ is reproduced without error from every $\left(x^{n}, y^{n}\right) \in \mathcal{A}_{n}^{*}$, we have

$$
\begin{aligned}
\mathcal{D}_{n} & =\bigcup_{\left(x^{n}, y^{n}\right) \in \mathcal{A}_{n}^{*}} \mathcal{D}_{n}\left(x^{n}, y^{n}\right) \\
& =\bigcup_{\left(x^{n}, y^{n}\right) \in \mathcal{A}_{n}^{*}} \varphi_{n}^{*-1}\left(x^{n}, y^{n}\right)
\end{aligned}
$$

where the second equality follows from (81). Furthermore, since $\varphi_{n}^{*}$ is deterministic, it is easy to see that

$$
\begin{aligned}
\varphi_{n}^{*-1}\left(x^{n}, y^{n}\right) \cap \varphi_{n}^{*-1}\left(\tilde{x}^{n}, \tilde{y}^{n}\right) & =\emptyset \\
\text { for all }\left(x^{n}, y^{n}\right) & \neq\left(\tilde{x}^{n}, \tilde{y}^{n}\right) .
\end{aligned}
$$

From (83) and (84), it is shown that $\left\{\varphi_{n}^{*-1}\left(x^{n}, y^{n}\right)\right\}_{\left(x^{n}, y^{n}\right) \in \mathcal{A}_{n}^{*}}$ is a partition of $\mathcal{D}_{n}$. Therefore, we have

$$
\begin{aligned}
1-P_{n}^{e} & =\sum_{\left(s^{n}, u^{n}\right) \in \mathcal{D}_{n}} P_{S^{n} U^{n}}\left(s^{n}, u^{n}\right) \\
& =\sum_{\left(x^{n}, y^{n}\right) \in \mathcal{A}_{n}^{*}} \sum_{\left(s^{n}, u^{n}\right) \in \varphi_{n}^{*-1}\left(x^{n}, y^{n}\right)} P_{S^{n} U^{n}}\left(s^{n}, u^{n}\right) \\
& =\sum_{\left(x^{n}, y^{n}\right) \in \mathcal{A}_{n}^{*}} P_{X^{n} Y^{n}}\left(x^{n}, y^{n}\right) \\
& =\operatorname{Pr}\left\{\left(X^{n}, Y^{n}\right) \in \mathcal{A}_{n}^{*}\right\}
\end{aligned}
$$

where the first equality comes from the definition of the decoding error probability and the third equality is due to the definition of $P_{X^{n} Y^{n}}(\cdot, \cdot)$, i.e., $P_{X^{n} Y^{n}}\left(x^{n}, y^{n}\right)=$ $\sum_{\left(s^{n}, u^{n}\right) \in \mathcal{S}^{n} \times \mathcal{U}^{n}: \varphi_{n}^{*}\left(s^{n}, u^{n}\right)=\left(x^{n}, y^{n}\right)} P_{S^{n} E^{n}}\left(s^{n}, u^{n}\right)$. Hence, we obtain ([D). It is easy to see from (I4) that $P_{n}^{e}$ satisfies (पI) i.e., the decoding error probability of $\psi_{n}^{*}$ in (ㅁ6) vanishes as $n$ goes to infinity.

In order to establish Theorem B, it remains to show that

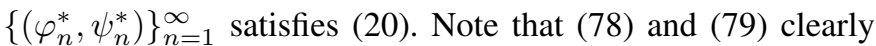
hold from (68) and ([0), respectively. To this end, we evaluate the success probability of the impersonation attack as follows:

$$
\begin{aligned}
P_{n}^{X} & =\max _{P_{\bar{X}^{n}}} \operatorname{Pr}\left\{\left(\bar{X}^{n}, Y^{n}\right) \in \mathcal{A}_{n}^{*}\right\} \\
& =\max _{P_{\bar{X}^{n}}} \sum_{\left(x^{n}, y^{n}\right) \in \mathcal{A}_{n}^{*}} P_{\bar{X}^{n}}\left(x^{n}\right) P_{Y^{n}}\left(y^{n}\right) \\
& \leq \max _{P_{\bar{X}^{n}}} \sum_{\left(x^{n}, y^{n}\right) \in \mathcal{A}_{n}^{*}} P_{\bar{X}^{n}}\left(x^{n}\right) \frac{P_{X^{n} Y^{n}}\left(x^{n}, y^{n}\right)}{P_{X^{n}}\left(x^{n}\right)} 2^{-n\left(\ell-\gamma_{n}\right)} \\
& \leq \max _{P_{\bar{X}^{n}}} \sum_{\substack{\left(x^{n}, y^{n}\right) \\
\in \mathcal{X}^{n} \times \mathcal{X}^{n}}} P_{\bar{X}^{n}}\left(x^{n}\right) \frac{P_{X^{n} Y^{n}}\left(x^{n}, y^{n}\right)}{P_{X^{n}}\left(x^{n}\right)} 2^{-n\left(\ell-\gamma_{n}\right)} \\
& =2^{-n\left(\ell-\gamma_{n}\right)}
\end{aligned}
$$

where the first inequality follows from ([13]) which implies

$$
\begin{aligned}
P_{Y^{n}}\left(y^{n}\right) & <\frac{P_{X^{n} Y^{n}}\left(x^{n}, y^{n}\right)}{P_{X^{n}}\left(x^{n}\right)} 2^{-n\left\{I(X ; Y)-\gamma_{n}\right\}} \\
& =\frac{P_{X^{n} Y^{n}}\left(x^{n}, y^{n}\right)}{P_{X^{n}}\left(x^{n}\right)} 2^{-n\left(\ell-\gamma_{n}\right)}
\end{aligned}
$$

for any $\left(x^{n}, y^{n}\right) \in \mathcal{A}_{n}^{*}$. Similarly, we have $P_{n}^{Y} \leq 2^{-n\left(\ell-\gamma_{n}\right)}$. Hence, we obtain (201) since $\lim _{n \rightarrow \infty} \gamma_{n}=0$.

Since Theorem B has been proved, we are now interested in a relation between the share rates and the correlation level attained by a pair $(f, g)$ of an encoder $f$ and a decoder $g$, which is given by the following claim:

Claim 6: Let $M$ and $M_{S}$ be arbitrary positive integers satisfying $M \geq M_{S}$. Then, there exists a pair $\left(f^{*}, g^{*}\right)$ of an encoder $f^{*}$ and a decoder $g^{*}$ for a $(2,2)$-threshold scheme with correlation level $\ell=\log M-H(S)$ in the non-asymptotic sense satisfying $|\mathcal{X}|=|\mathcal{Y}|=|\mathcal{U}|=M$ and $|\mathcal{S}|=M_{S}$.

Remark 5: According to Claim 6, the rates of shares and randomness are $\log |\mathcal{X}|=\log |\mathcal{Y}|=\log |\mathcal{U}|=\log M=$ $H(S)-\ell$, which coincides with the lower bounds of the

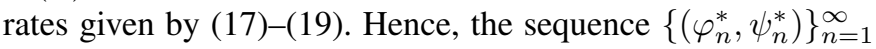
defined by (72) and (176) also achieves all the bounds in 
Theorem [. Observe that the sequence of encoders $\left\{\varphi_{n}^{*}\right\}_{n=1}^{\infty}$ in this section is simpler than the sequence of encoders presented in Theorems 2. For instance, $S^{n}$ cannot be encoded symbolwisely by the sequence of encoders in the proof of Theorem $\square$ since the correlation of two shares is generated by the random variable $U_{n}^{\mathcal{L}}$ in both shares contained in common. On the other hand, symbolwise encoding is possible by the sequence of encoders in this section since $X_{i}$ and $Y_{i}$ are correlated due to $f$ for every $i=1,2, \ldots, n$. Furthermore, such symbolwise encoding also enables us that $I\left(S_{i} ; X_{i}\right)=$ $I\left(S_{i} ; Y_{i}\right)=0$ for every $i=1,2, \ldots, n$, which is stronger than the security condition given by (III) in Theorem $\square$.

However, we note that $M, M_{S}$ and the correlation level $\ell$ cannot be set arbitrarily in Claim 6 although they can be taken arbitrarily in Theorem 乙, which is compensation for the simplicity.

Remark 6: In the threshold scheme with detectability of substitution attacks in a non-asymptotic setup (e.g., [3]-[8]), it is shown that any ideal secret sharing scheme cannot detect any forgery of shares with probability 1 . Furthermore, as is shown in [26], we note that the ideal secret sharing schemes can be realized if and only if $|\mathcal{X}|=|\mathcal{Y}|=|\mathcal{S}|$ and $S$ is uniformly distributed.

Similarly, in the asymptotic setup discussed in this section, it is impossible for any $(f, g)$ of an ideal $(2,2)$-threshold scheme to achieve $P_{n}^{X}$ and $P_{n}^{Y}$ with exponential order of $n$ because the correlation level $\log M-H(S)=0$ is satisfied if and only if $M=|\mathcal{S}|$ and $S$ is uniformly distributed. On the other hand, we note that $\ell$ is positive for arbitrary distribution of $S$ if $\min \{|\mathcal{X}|,|\mathcal{Y}|,|\mathcal{U}|\}>|\mathcal{S}|$.

Proof of Claim 6: From (पII), let us define

$$
\begin{aligned}
\mathcal{X} & =\mathcal{Y}=\mathcal{U}=\{0,1, \ldots, M-1\} \\
\mathcal{S} & =\left\{0,1, \ldots, M_{S}-1\right\}
\end{aligned}
$$

where $M \geq M_{S}$. Define the encoder $f^{*}: \mathcal{S} \times \mathcal{U} \rightarrow \mathcal{X} \times \mathcal{Y}$ for a secret $s \in \mathcal{S}$ and a random number $u \in \mathcal{U}$ as

$$
f^{*}(s, u)=(s \ominus u, u)
$$

where $\ominus$ denotes the subtraction of modulo $M$. Then, the corresponding decoder $g^{*}: \mathcal{X} \times \mathcal{Y} \rightarrow \mathcal{S} \cup\{\lambda\}$ can be written as

$$
g^{*}(x, y)=\left\{\begin{array}{cl}
x \oplus y, & \text { if } x \oplus y \in \mathcal{S} \\
\lambda, & \text { otherwise }
\end{array}\right.
$$

where $\oplus$ represents the addition of modulo $M$. Note that the secret $s$ can be decoded by $g^{*}$ without error, and hence, (69) is satisfied. Furthermore, we can check that a pair of the shares $(X, Y)$ is generated according to the conditional probability distribution

$$
P_{X Y \mid S}(x, y \mid s)=\left\{\begin{array}{cl}
1 / M, & \text { if } s=x \oplus y \in \mathcal{S} \\
0, & \text { otherwise }
\end{array}\right.
$$

if we apply the encoder $f^{*}$ defined in (901) to the secret $S$ with an arbitrary probability distribution $P_{S}(\cdot)$. Hence, the following discussion holds for an arbitrary distribution on $S$.
This idea is based on the secret sharing scheme for nonuniform secret distribution studied in [26].

We show that (68) is satisfied by $X$ and $Y$ generated by $f^{*}$. For every fixed $x \in \mathcal{X}$ and $s \in \mathcal{S}$, we can check that there exists a unique $y \in \mathcal{Y}$, satisfying $s=g^{*}(x, y)$. Hence, it holds from (92) that

$$
\begin{aligned}
P_{X \mid S}(x \mid s) & =\sum_{y \in \mathcal{Y}} P_{X Y \mid S}(x, y \mid s) \\
& =\frac{1}{M}
\end{aligned}
$$

for every $(x, s) \in \mathcal{X} \times \mathcal{S}$. Then, we have

$$
\begin{aligned}
P_{X}(x) & =\sum_{s \in \mathcal{S}} P_{X \mid S}(x \mid s) P_{S}(s) \\
& =\sum_{s \in \mathcal{S}} \frac{1}{M} \cdot P_{S}(s) \\
& =\frac{1}{M} .
\end{aligned}
$$

From (293) and (94), it is shown that $S$ and $X$ are statistically independent. Similarly, it can be shown that $S$ and $Y$ are statistically independent, and hence, (68) is proved.

The correlation level of $X$ and $Y$ generated by $f^{*}$ can be calculated as follows. We note that

$$
\begin{aligned}
H(X Y) & \stackrel{(\mathrm{e})}{=} H(U S) \\
& \stackrel{(\mathrm{f})}{=} H(U)+H(S) \\
& =\log M+H(S)
\end{aligned}
$$

where the marked equalities (e) and (f) hold since

(e) there exists a bijection between $\mathcal{U} \times \mathcal{S}$ and $\mathcal{X} \times \mathcal{Y}$.

(f) $U$ and $S$ are statistically independent.

Therefore, we obtain from (24) and (25) that

$$
\begin{aligned}
I(X ; Y) & =H(X)+H(Y)-H(X Y) \\
& =2 \log M-\{\log M+H(S)\} \\
& =\log M-H(S) .
\end{aligned}
$$

Hence, it is shown that the pair $\left(f^{*}, g^{*}\right)$ of the encoder and the decoder actually realizes a $(2,2)$-threshold scheme with correlation level $\log M-H(S)$.

\section{CONCLUSION}

This paper is concerned with coding theorems for a $(2,2)$ threshold scheme in the presence of an opponent who impersonates one of the participants. We have considered an asymptotic setup of the $(2,2)$-threshold scheme in which $n$ secrets from a memoryless source are encoded to two shares by using a uniform random number, and the two shares are decoded to the $n$ secrets with permitting negligible decoding error probability. We have investigated the minimum attainable rates of the two shares and the uniform random number, and the maximum exponents of the probabilities of the successful impersonation from a Shannon-theoretic viewpoint. We have presented coding theorems for two cases of encoding, i.e., blockwise and symbolwise encoding.

In the first case, we have considered the situation where the $n$ secrets are encoded blockwisely to two shares. We 
have defined the correlation level $\ell \geq 0$ of the shares as the limit of the normalized mutual information between the two shares. In the converse part it is shown that for any sequence $\left\{\left(\varphi_{n}, \psi_{n}\right)\right\}_{n=1}^{\infty}$ of pairs of an encoder $\varphi_{n}$ and a decoder $\psi_{n}$ that asymptotically realizes a $(2,2)$-threshold scheme with the correlation level $\ell$, none of the rates can be less than $H(S)+\ell$, where $H(S)$ denotes the entropy of the source, and the exponent of the probability of the successful impersonation cannot be less than $\ell$. In addition, we have shown the existence of a sequence $\left\{\left(\varphi_{n}^{*}, \psi_{n}^{*}\right)\right\}_{n=1}^{\infty}$ of pairs of an encoder $\varphi_{n}^{*}$ and a decoder $\psi_{n}^{*}$ that attains all the bounds given in the converse part. The obtained results can be easily extended to the case where the $n$ secrets are generated from a stationary ergodic source.

In the second case, we have considered the situation where the $n$ secrets are encoded symbolwisely to two shares of length $n$ by repeatedly applying the encoder of an ordinary $(2,2)-$ threshold scheme to the $n$ secrets. While the above converse part is valid in this setup, we can give another interesting decoder in the direct part. That is, we have shown that the impersonation by an opponent can be verified with probability close to one by verifying the joint typicality of the two shares. It turns out that these encoder and decoder also attain all the bounds in the converse part.

\section{ACKNOWLEDGMENT}

The authors would like to thank Prof. H. Nagaoka in the University of Electro-Communications, for his helpful comments.

\section{REFERENCES}

[1] A. Shamir, "How to share a secret," Communications of the ACM, vol. 22, no. 11, pp. 612-613, 1979.

[2] G. R. Blakley, "Safeguarding cryptographic keys," AFIPS 1979 National Computer Conference, vol. 48, pp. 313-317, 1979.

[3] R. J. McEliece and D. V. Sarwate, "On sharing secrets and Reed Solomon codes," Communications of the ACM, vol. 24, no. 9, pp. 583$584,1981$.

[4] E. D. Karnin, J. W. Greene, and M. E. Hellman, "On secret sharing systems," IEEE Trans. Inform. Theory, vol. 29, no. 1, pp. 35-41, 1983.

[5] M. Tompa and H. Woll, "How to share a secret with cheaters," Journal of Cryptology, vol. 1, no. 3, pp. 133-138, 1988. Preliminary version: CRYPTO'86, LNCS 263, pp.261-265.

[6] M. Carpentieri, A. D. Santis, and U. Vaccaro, "Size of shares and probability of cheating in threshold scheme," Advances in CryptologyEUROCRYPT'93, LNCS 765, Springer-Verlag, pp. 118-125, 1994.

[7] K. Kurosawa, S. Obana, and W. Ogata, " $t$-cheater identifiable $(k, n)$ secret sharing schemes," Advances in Cryptology-CRYPTO'95, LNCS 963, Springer-Verlag, pp. 410-423, 1995.

[8] W. Ogata, K. Kurosawa, and D. R. Stinson, "Optimum secret sharing scheme secure against cheating," SIAM Journal of Discrete Mathematics, vol. 20, no. 1, pp. 79-95, 2006. Preliminary version: EUROCRYPT'96, LNCS 1070, pp.200-211.

[9] S. Obana and T. Araki, "Secret sharing schemes secure against cheating for arbitrary secret distribution," Advances in Cryptology-ASIACRYPT 2006, LNCS 4284, Springer-Verlag, pp. 364-379, 2006.

[10] G. J. Simmons, "Authentication theory/coding theory," Advances in Cryptology-CRYPTO'84, LNCS 196, Springer-Verlag, pp. 411-431, 1985.

[11] H. Yamamoto, "On secret sharing communication systems with two or three channels," IEEE Trans. Information Theory, vol. 32, no. 3, pp. 387393, 1986.

[12] H. Koga, "Coding theorems on the threshold secret sharing scheme for a general source," IEEE Trans. Information Theory, vol. 54, no. 6, pp. $2658-2677,2006$
[13] D. R. Stinson and S. A. Vanstone, "A combinatorial approach to threshold schemes," SIAM J. on Discrete Math., no. 1, pp. 230-237, 1988. Preliminary version: CRYPTO'87, pp.331-339.

[14] H. Koga and H. Yamamoto, "Coding theorems for secret-key authentication systems," IEICE Trans. Fundamentals, vol. E83-A, no. 8, pp. 1691$1703,2000$.

[15] H. Koga, "A generalization of the Simmons' bounds on secret-key authentication systems," IEICE Trans. Fundamentals, vol. E83-A, no. 10, pp. 1983-1985, 2000 .

[16] U. M. Maurer, "Authentication theory and hypothesis testing," IEEE Trans. on Information Theory, vol. 46, no. 4, pp. 1350-1356, 2000. Preliminary version: STACS'96, LNCS 1046, pp.387-398, 1996.

[17] C. E. Shannon, "Communication theory of secrecy systems," Bell Tech. J., vol. 28, pp. 656-715, Oct. 1949.

[18] M. E. Hellman, "An extension of the Shannon theory approach to cryptography," IEEE Trans. Information Theory, vol. 23, no. 3, pp. 289294, 1977.

[19] H. Yamamoto, "Coding theorems for Shannon's cipher system with correlated source outputs, and common information," IEEE Trans. Information Theory, vol. 40, no. 1, pp. 85-95, 1994.

[20] H. Yamamoto, "Information theory in cryptography," IEICE Trans. Fundamentals, vol. E-74, no. 9, pp. 2456-2464, 1991.

[21] U. M. Maurer, "Secret key agreement by public discussion based on common information," IEEE Trans. Information Theory, vol. 39, no. 3, pp. 733-743, 1993.

[22] R. Ahlswede and I. Csiszár, "Common randomness in information theory and cryptography- part I: secret sharing," IEEE Trans. Information Theory, vol. 39, no. 4, pp. 1121-1132, 1993.

[23] T. M. Cover and J. A. Thomas, Elements of Information Theory. Wiley and Interscience, second ed., 2006.

[24] R. E. Blahut, Principles and Practice of Information Theory. Addison Wesley, 1991.

[25] T. S. Han, Information-Spectrum Methods in Information Theory. Springer-Verlag, 2003.

[26] C. Blundo, A. D. Santis, and U. Vaccaro, "On secret sharing schemes," Information Processing Letters, no. 65, pp. 25-32, 1998.

Mitsugu Iwamoto (S'01-M'04) received the B.E., the M.E., and Ph.D degrees from the University of Tokyo, Japan, in 1999, 2001, and 2004, respectively. In 2004, he joined University of Electro-Communications, where he is currently an Assistant Professor of the Center for Frontier Science and Engineering. His research interests include information theory and cryptography. He is a member of IACR and IEICE.

Hiroki Koga (S'93-M'94) received B.E., M.E. and Ph.D degrees from the University of Tokyo, in 1990, 1992 and 1995, respectively.

From 1995 to 1999, he was a Research Associate in Graduate school of Engineering, the University of Tokyo. Since 1999, he has been with the University of Tsukuba, where he is currently an Associate Professor of Graduate School of Systems and Information Engineering. His research interests are in Shannon theory and information security. $\mathrm{He}$ is a senior member of the IEICE.

Hirosuke Yamamoto (S'77-M'80-SM'03-F'11) received the B.E. degree from Shizuoka University, Shizuoka, Japan, in 1975 and the M.E. and Ph.D. degrees from the University of Tokyo, Tokyo, Japan, in 1977 and 1980, respectively, all in electrical engineering. In 1980, he joined Tokushima University. He was an Associate Professor at Tokushima University from 1983 to 1987, the University of Electro-Communications from 1987 to 1993, and the University of Tokyo from 1993 to 1999. Since 1999, he has been a Professor at the University of Tokyo and is currently with the Department of Complexity Science and Engineering at the university. In 1989-1990, he was a Visiting Scholar at the Information Systems Laboratory, Stanford University, Stanford, CA. His research interests are in Shannon theory, data compression algorithms and cryptology.

Dr. Yamamoto served as the Chair of IEEE Information Theory Society Japan Chapter in 2002-2003, the TPC Co-Chair of the ISITA2004, the TPC Chair of the ISITA2008, the president of the SITA (Society of Information Theory and its Applications) in 2008-2009, an Associate Editor for Shannon Theory, the IEEE Transactions on Information Theory in 2007-2010, Editor-in-Chief for the IEICE Transactions on Fundamentals of Electronics, Communications and Computer Sciences in 2009-2011. He is a Fellow of the IEICE and he is currently the President of the Engineering Sciences Society, IEICE. 